\title{
Tube Formulas and Complex Dimensions of Self-Similar Tilings
}

\author{
Michel L. Lapidus • Erin P.J. Pearse
}

Received: 3 February 2009 / Accepted: 21 January 2010 / Published online: 25 June 2010

(C) Springer Science+Business Media B.V. 2010

\begin{abstract}
We use the self-similar tilings constructed in (Pearse in Indiana Univ. Math J. 56(6):3151-3169, 2007) to define a generating function for the geometry of a self-similar set in Euclidean space. This tubular zeta function encodes scaling and curvature properties related to the complement of the fractal set, and the associated system of mappings. This allows one to obtain the complex dimensions of the self-similar tiling as the poles of the tubular zeta function and hence develop a tube formula for self-similar tilings in $\mathbb{R}^{d}$. The resulting power series in $\varepsilon$ is a fractal extension of Steiner's classical tube formula for convex bodies $K \subseteq \mathbb{R}^{d}$. Our sum has coefficients related to the curvatures of the tiling, and contains terms for each integer $i=0,1, \ldots, d-1$, just as Steiner's does. However, our formula also contains a term for each complex dimension. This provides further justification for the term "complex dimension". It also extends several aspects of the theory of fractal strings to higher dimensions and sheds new light on the tube formula for fractals strings obtained in (Lapidus and van Frankenhuijsen in Fractal Geometry, Complex Dimensions and Zeta Functions: Geometry and Spectra of Fractal Strings, 2006).
\end{abstract}

The work of MLL was partially supported by the US National Science Foundation under the research grants DMS-0070497 and DMS-0707524. The work of EPJP was partially supported by the University of Iowa Department of Mathematics NSF VIGRE grant DMS-0602242.

M.L. Lapidus

Department of Mathematics, University of California, Riverside, CA 92521-0135, USA

e-mail: lapidus@math.ucr.edu

url: http://www.math.ucr.edu/ lapidus

E.P.J. Pearse ( $\varangle)$

Department of Mathematics, Cornell University, Ithaca, NY 14853-4201, USA

e-mail: erin@math.cornell.edu

url: http://www.math.uiowa.edu/ epearse/

Present address:

E.P.J. Pearse

University of Iowa, Iowa City, IA 52242-1419, USA

e-mail: erin-pearse@uiowa.edu 
Keywords Complex dimensions - Zeta functions - Tube formula · Fractal Steiner formula Inradius · Self-similar tiling · Curvature matrix · Generating function for the geometry · Distributional explicit formula $\cdot$ Fractal string

Mathematics Subject Classification (2000) Primary 11M41 - 28A12 - 28A75 - 28A80 . $52 \mathrm{~A} 39 \cdot 52 \mathrm{C} 07 \cdot$ Secondary $11 \mathrm{M} 36 \cdot 28 \mathrm{~A} 78 \cdot 28 \mathrm{D} 20 \cdot 42 \mathrm{~A} 16 \cdot 42 \mathrm{~A} 75 \cdot 52 \mathrm{~A} 20 \cdot 52 \mathrm{~A} 38$

\section{Introduction}

The main result of this paper is a tube formula for a certain class of fractal sets. Here, a tube formula for a bounded set $A \subseteq \mathbb{R}^{d}$ is an explicit expression for the $d$-dimensional volume of the inner $\varepsilon$-neighborhood of $A$, i.e.,

$$
V_{A}(\varepsilon)=\operatorname{vol}_{d}\{x \in A \vdots \operatorname{dist}(x, \partial A) \leq \varepsilon\} .
$$

Such formulas have myriad applications to geometry and have roots in the results of Steiner (when $A$ is convex) and Weyl (when $A$ is a smooth manifold). In order to explain how our result is an extension of these classical formulas, and how it is related to the development of a notion of curvature for fractal sets, we give a brief encapsulation of Steiner's theorem. Here, the Minkowski sum of two subsets $A$ and $B$ of $\mathbb{R}^{d}$ is denoted by $A+B=\left\{x \in \mathbb{R}^{d}: x=\right.$ $a+b$ for $a \in A, b \in B\}$. Then, if $B^{k}$ is the closed $k$-dimensional unit ball in $\mathbb{R}^{d}$, one can denote the outer $\varepsilon$-neighborhood of a set $A \subseteq \mathbb{R}^{d}$ by

$$
A+\varepsilon B^{d}=\left\{x \in \mathbb{R}^{d}: \operatorname{dist}(x, A) \leq \varepsilon\right\} .
$$

Theorem 1.1 (Steiner's formula) If $A \subseteq \mathbb{R}^{d}$ is convex and compact, then the d-dimensional volume of $A+\varepsilon B^{d}$ is given by

$$
\operatorname{vol}_{d}\left(A+\varepsilon B^{d}\right)=\sum_{k=0}^{d} \mu_{k}(A) \operatorname{vol}_{d-k}\left(B^{d-k}\right) \varepsilon^{d-k},
$$

where $\mu_{k}$ is the renormalized $k$-dimensional intrinsic volume.

Note that this formula is simply a polynomial in $\varepsilon$; the coefficients are constants determined by the curvature of $A$ (and the unit ball). Up to some normalizing constant, the $k$-dimensional intrinsic volume is the same thing as the $(d-k)^{\text {th }}$ Quermassintegral (from Minkowski's theory of mixed volumes). The valuation $\mu_{k}$ can be defined via integral geometry as the average measure of orthogonal projections to $(d-k)$-dimensional subspaces; see [16, Chap. 7]. For now, we note that (up to a multiplicative constant), there is a correspondence

$$
\begin{array}{lll}
\mu_{0} \sim \text { Euler characteristic }, & & \mu_{d-1} \sim \text { surface area, } \\
\mu_{1} \sim \text { mean width }, & & \mu_{d} \sim \text { volume },
\end{array}
$$

see [37, Sect. 4.2] for further details.

When $A$ is sufficiently regular (i.e., when its boundary is a $C^{2}$ surface), these coefficients can be given in terms of curvature tensors, and in fact Steiner's tube formula coincides with the one obtained by Weyl in [40]. In [4], Federer unified the tube formulas of Steiner (for convex bodies, as described in [37, Chap. 4]) and of Weyl (for smooth submanifolds, 
as described in [9] and [40]) and extended these results to sets of positive reach. ${ }^{1}$ It is worth noting that Weyl's tube formula for smooth submanifolds of $\mathbb{R}^{d}$ is expressed as a polynomial in $\varepsilon$ with coefficients defined in terms of curvatures (in the classical sense) that are intrinsic to the submanifold [40]. See [1, Sects. 6.6-6.9] and the book [9]. Federer's tube formula has since been extended in various directions by a number of researchers in integral geometry and geometric measure theory, including [6, 7, 36-38, 42, 43], and most recently (and most generally) in [13]. The books [9] and [37] contain extensive endnotes with further information and many other references.

Note that (1.2) gives the volume of the set of points which are within $\varepsilon$ of $A$, including the points of $A$. If we denote the exterior $\varepsilon$-neighborhood of $A$ by

$$
A_{\varepsilon}^{e x t}:=\left\{x \in \mathbb{R}^{d} \backslash A \vdots \operatorname{dist}(x, A) \leq \varepsilon\right\}
$$

then it is immediately clear that omitting the $d^{\text {th }}$ term gives

$$
\operatorname{vol}_{d}\left(A_{\varepsilon}^{e x t}\right)=\sum_{k=0}^{d-1} C_{k}(A) \varepsilon^{d-k}
$$

with $C_{k}(A)=\mu_{k}(A) \operatorname{vol}_{d-k}\left(B^{d-k}\right)$. These coefficients $C_{k}(A)$ are called the total curvatures of $A$ and they are key geometric invariants. Even more importantly, $C_{k}(A)$ can be localized and understood as the curvature measures described in [4] and [37, Chap. 4]. In this case, for a Borel set $\beta \subseteq \mathbb{R}^{d}$, one has

$$
\operatorname{vol}_{d}\left\{x \in A_{\varepsilon}^{e x t}: p(x, A) \in \beta\right\}=\sum_{k=0}^{d-1} C_{k}(A, \beta) \varepsilon^{d-k}
$$

where $p(x, A)$ is the metric projection of $x$ to $A$, that is, the closest point of $A$ to $x$. In fact, the curvature measures are obtained axiomatically in [37] as the coefficients of the tube formula, and it is this approach that we hope to emulate in a forthcoming work, based on a localized version of the tube formula obtained in the present paper. In other words, we believe that $\kappa_{k}$ (introduced in (1.7) below) may also be understood as a (total) curvature, in a suitable sense, and we expect that $\kappa_{k}$ can be localized as a curvature measure (or rather, current). A more rigorous formulation of these ideas is currently underway in [23].

Our Main Result Since it is somewhat arduous to express our tube formula with complete precision, we present a somewhat simplified version of it here, so that the key features are not overshadowed by technical details (the exact hypotheses necessary for this formulation are given in Corollary 8.7).

Theorem 1.2 The d-dimensional volume of the tubular neighborhood of a sufficiently nice self-similar fractal set $F$ is given by the following distributional explicit formula:

$$
V_{F}(\varepsilon)=\sum_{\omega \in \mathcal{D}_{\mathfrak{s}} \cup\{0,1, \ldots, d-1\}} c_{\omega} \varepsilon^{d-\omega},
$$

\footnotetext{
${ }^{1}$ A set $A$ has positive reach iff there is some $\delta>0$ such that any point $x$ within $\delta$ of $A$ has a unique metric projection to $A$, i.e., that there is a unique point $A$ minimizing $\operatorname{dist}(x, A)$. Equivalently, every point $q$ on the boundary of $A$ lies on a sphere of radius $\delta$ which intersects $\partial A$ only at $q$.
} 
where for each fixed $\omega \in \mathcal{D}_{\mathfrak{s}}$,

$$
c_{\omega}:=\operatorname{res}\left(\zeta_{\mathfrak{s}}(s) ; \omega\right) \sum_{k=0}^{d} \frac{g^{\omega-k}}{\omega-k} \kappa_{k} .
$$

Here, $\zeta_{\mathfrak{s}}$ is a zeta function which encodes scaling information about $F, \mathcal{D}_{\mathfrak{s}}$ is the set of poles of $\zeta_{\mathfrak{s}}$ (the complex dimensions ${ }^{2}$ ), and $g$ and $\kappa_{k}$ are geometric data obtained from the complement of $F$. Roughly speaking, $g$ is the size of the largest bounded component of $\mathbb{R}^{d} \backslash F$ (the generator), and $\kappa_{k}$ are "curvatures" of the same set, in some sense. Also, "sufficiently nice" requires that the open set condition is satisfied for a set whose boundary is contained in $F$; cf. [34, Theorem 6.2].

Theorem 1.2 is a special case of a more fundamental result which is valid under much more general hypotheses: the tube formula for fractal sprays given in Theorem 7.4:

Theorem 1.3 The d-dimensional volume of the inner tubular neighborhood of a fractal spray $\mathcal{T}$ with a generator $G$ is given by the following distributional explicit formula:

$$
V_{\mathcal{T}}(\varepsilon)=\sum_{\omega \in \mathcal{D}_{\mathcal{T}}} \operatorname{res}\left(\zeta_{\mathcal{T}}(\varepsilon, s) ; \omega\right)
$$

where

$$
\zeta_{\mathcal{T}}(\varepsilon, s):=\varepsilon^{d-s} \zeta_{\mathfrak{s}}(s) \sum_{k=0}^{d} \frac{g^{s-k}}{s-k} \kappa_{k}
$$

and $\mathcal{D}_{\mathcal{T}}:=\mathcal{D}_{\mathfrak{s}} \cup\{0,1, \ldots, d-1\}$ is the set of poles of the tubular zeta function $\zeta_{\mathcal{T}}$.

In (1.9), the numbers $\kappa_{0}, \kappa_{1}, \ldots, \kappa_{d}$ refer to a chosen "Steiner-like representation" of $G$ (an expression of the inner tube for $G$ which satisfies certain very mild conditions discussed in Definition 5.1).

Our tube formula extends previous results in two ways. On one hand, it provides a fractal analogue of the classical Steiner formula of convex geometry. On the other hand, the tube formula (1.8) also provides a natural higher-dimensional analogue of the tube formula for fractal strings obtained in [30] and recalled in (6.16). The present work can be considered as a further step towards a higher-dimensional theory of fractal strings and their complex dimensions, especially in the self-similar case, following upon [27, Sects. 10.2 and 10.3], and our earlier papers [21, 33]. This is discussed further in Sect. 8.2 and in Remark 9.1.

To emphasize the present analogy with (1.6), consider that, with the obvious change of notation, Steiner's formula (1.4) may be rewritten

$$
\operatorname{vol}_{d}\left(A_{\varepsilon}^{e x t}\right)=\sum_{k \in\{0,1, \ldots, d-1\}} c_{k} \varepsilon^{d-k} .
$$

The obvious similarities between the tube formulas (1.6) and (1.10) is striking. Our tube formula is a "fractal power series" in $\varepsilon$, rather than just a polynomial in $\varepsilon$ as in Steiner's (and Weyl's) formula. Moreover, our series is summed not just over the 'integral dimensions'

\footnotetext{
${ }^{2}$ Actually, elsewhere in this paper, complex dimensions refers to the set $\mathcal{D}_{\mathcal{T}}=\mathcal{D}_{\mathfrak{s}} \cup\{0,1, \ldots, d-1\}$ (the poles of $\zeta_{\mathcal{T}}$ ) and we refer to $\mathcal{D}_{\mathfrak{s}}$ as the scaling complex dimensions; see Definition 7.3.
} 
$\{0,1, \ldots, d-1\}$, but also over the countable set $\mathcal{D}_{\mathfrak{s}}$ of complex dimensions. The coefficients $c_{\omega}$ of the tube formula are expressed in terms of the 'curvatures' and the inradii of the generators of the tiling. It is intriguing to consider that the extra terms appearing in our formula are oscillatory, as evinced by the purely imaginary components of the complex dimensions. Moreover, fractals are objects with (multiplicative) geometric oscillations, in the sense that the same geometric patterns repeat, at different scales of magnification. This is the essential theme of the theory of complex dimensions as expounded in [30].

The primary object of study in [30] is a fractal string, a countable collection $L=\left\{L_{j}\right\}_{j=1}^{\infty}$ of disjoint open intervals which form a bounded open subset of $\mathbb{R}$. Due to the trivial geometry of such intervals, this reduces to studying the lengths of these intervals $\mathcal{L}=\left\{\ell_{j}\right\}_{j=1}^{\infty}$, and the sequence $\mathcal{L}$ is also referred to as a fractal string. The tube formula for a fractal string $\mathcal{L}$ (and in particular, for a self-similar tiling in $\mathbb{R}$ ) is defined to be $V_{\mathcal{L}}(\varepsilon):=V_{L}(\varepsilon)$ and is shown to be essentially given by a sum of the form

$$
V_{\mathcal{L}}(\varepsilon)=\sum_{\omega \in \mathcal{D}_{\mathcal{L}} \cup\{0\}} c_{\omega} \varepsilon^{1-\omega}
$$

in [30], Theorem 8.1. Here, the sum is taken over the set of complex dimensions $\mathcal{D}_{\mathcal{L}}=$ \{poles of $\zeta_{\mathcal{L}}$, and $c_{\omega}$ is given in terms of the residue of $\zeta_{\mathcal{L}}(s)$ at $s=\omega$, the geometric zeta function of $\mathcal{L}$ (defined as the meromorphic continuation of the Dirichlet series $\sum_{j=1}^{\infty} \ell_{j}^{s}$, for $s \in \mathbb{C})$. The definition $V_{\mathcal{L}}(\varepsilon):=V_{L}(\varepsilon)$ is justified because, as is shown in [25], $V_{L}$ depends exclusively on $\mathcal{L}$.

In [30, Sect. 1.4] (following [26]), a fractal spray is defined to be given by a nonempty bounded open set $G \subseteq \mathbb{R}^{d}$ called the generator (or "basic shape" in [30]), scaled by a fractal string $\mathcal{L}=\left\{\ell_{j}\right\}_{j=1}^{\infty}$. That is, a fractal spray is a bounded open subset of $\mathbb{R}^{d}$ which is the disjoint union of open sets $\omega_{j}$ for $j=1,2, \ldots$, where $\omega_{j}$ is congruent to $\ell_{j} G$ (the homothetic of $\omega$ by $\ell_{j}$ ) for each $\ell_{j}$. Thus, a fractal string is a fractal spray on the generator $G=(0,1)$, the unit interval. In the context of the current paper, a self-similar tiling is a union of fractal sprays on the generators $G_{1}, \ldots, G_{Q}$, each scaled by a fixed self-similar string. In fact, we first prove Theorem 1.3 for the more general case of fractal sprays, and then refine it to obtain the formula for self-similar tilings.

For fractal sets, the tube formula may also be used to assess the Minkowski measurability and to determine the Minkowski dimension (also called the box or box-counting dimension) and the value of the Minkowski content (when it exists); see Corollary 8.5 and Remark 8.6. Minkowski dimension of the boundary $\partial A$ of a bounded open subset $A \subseteq \mathbb{R}^{d}$ is a real-valued extension of the usual (topological) notion of dimension defined by

$$
D=\operatorname{dim}_{\mathcal{M}}(\partial A):=\inf \left\{\alpha \geq 0: V_{A}(\varepsilon)=O\left(\varepsilon^{1-\alpha}\right) \text { as } \varepsilon \rightarrow 0^{+}\right\} .
$$

Minkowski dimension frequently coincides with Hausdorff dimension (for example, for fractal sets defined in terms of an iterated function system with mappings that do not overlap too much) and in general, $\operatorname{dim}_{\mathcal{H}}(X) \leq \operatorname{dim}_{\mathcal{M}}(X)$. The set $\partial A$ is said to be Minkowski measurable, with Minkowski content

$$
\mathcal{M}(\partial A):=\lim _{\varepsilon \rightarrow 0^{+}} V_{A}(\varepsilon) \varepsilon^{-\left(d-\operatorname{dim}_{\mathcal{M}}(\partial A)\right)},
$$

if this limit exists and is both finite and strictly positive.

Further (potential) applications and extensions of our results are discussed in Sect. 10 and elsewhere in the paper; in particular, please see Remark 8.12. Some of the main results of this paper were announced in [22]. 
Strategy for Obtaining the Tube Formula For simplicity, we limit ourselves here to the self-similar case. In [32], the second author has shown that a certain (self-similar) tiling $\mathcal{T}$ is canonically associated with any self-similar set. This tiling is defined via the finite collection $\Phi=\left\{\Phi_{j}\right\}_{j=1}^{J}$ of contractive similarity transformations which defines the self-similar set. This tiling $\mathcal{T}$ is essentially a decomposition of the complement of the unique self-similar set associated with $\Phi$, and is reviewed in greater detail in Sect. 2. Several conditions are given in [34] which describe precisely when the tube formula of a self-similar set may be obtained from a tube formula for the self-similar tiling $\mathcal{T}$. The results of the present study allow one to obtain a tube formula for the (large) class of fractal sets satisfying the "compatibility conditions" of [34, Theorem 4.4 or Theorem 6.2].

At the heart of this paper is the tubular zeta function $\zeta_{\mathcal{T}}(s)$ of a self-similar tiling $\mathcal{T}$. It will take some work before we are able to describe this meromorphic distribution-valued function precisely in Sect. 7. The function $\zeta_{\mathcal{T}}$ is a generating function for the geometry of a self-similar tiling: it encodes the density of geometric states of a tiling, including curvature and scaling properties. The poles of $\zeta_{\mathcal{T}}$ are the complex dimensions $\mathcal{D}_{\mathcal{T}}$ of the tiling, and we obtain a tube formula for $\mathcal{T}$ given as a sum over $\mathcal{D}_{\mathcal{T}}$ of the residues of $\zeta_{\mathcal{T}}$, taken at the complex dimensions. The complex dimensions generalize the Minkowski dimension in the sense that, for any tiling $\mathcal{T}, \sup \left\{\mathcal{D}_{\mathcal{T}} \cap \mathbb{R}\right\}$ is equal to the Minkowski dimension of the underlying self-similar set $F$.

The first ingredient of $\zeta_{\mathcal{T}}$ is a scaling zeta function $\zeta_{\mathfrak{s}}(s)$ which encodes the scaling properties of the tiling and is discussed in Sect. 4.2. This comparatively simple zeta function is the Mellin transform of a discrete scaling measure $\eta_{\mathfrak{s}}$ which encodes the combinatorics of the scaling ratios of a self-similar tiling. More precisely, if one considers a composition of similarity mappings $\Phi_{j}$, each with scaling ratio $r_{j}$, for $j=1, \ldots, J$, then

$$
\Phi_{w}=\Phi_{w_{1} w_{2} \ldots w_{n}}=\Phi_{w_{n}} \circ \cdots \circ \Phi_{w_{2}} \circ \Phi_{w_{1}}
$$

has scaling ratio $r_{w}=r_{w_{1}} r_{w_{2}} \cdots r_{w_{n}}$, where $w_{i} \in\{1,2, \ldots, J\}$. The measure $\eta_{\mathfrak{s}}$ is a sum of Dirac masses, where each mass is located at a reciprocal scaling ratio $r_{w}^{-1}$. The total mass of any point in the support of $\eta_{\mathfrak{s}}$ corresponds to the multiplicity with which such a scaling ratio can occur. The scaling zeta function $\zeta_{\mathfrak{s}}$ is formally identical to the zeta functions studied in [30]. The function $\zeta_{\mathfrak{s}}$ also allows us to define the scaling complex dimensions of a selfsimilar set in $\mathbb{R}^{d}$ (as the poles of $\zeta_{\mathfrak{s}}$ ), and we find these dimensions to have the same structure as in the 1-dimensional case; see Sect. 4.3 and Remark 8.6. The definition and properties of the scaling measure $\eta_{\mathfrak{s}}$ and zeta function $\zeta_{\mathfrak{s}}$ is the subject of Sect. 4.1.

The next ingredient of $\zeta_{\mathcal{T}}$ is a generator tube formula $\gamma_{G}$. In [32], it is shown that certain tiles $G_{1}, \ldots, G_{Q}$ of $\mathcal{T}$ are generators in the sense that any tile $R_{n}$ of $\mathcal{T}$ is the image of some $G_{q}$ under some composition of the mappings $\Phi_{j}$, i.e.,

$$
R_{n} \in \mathcal{T} \quad \Longrightarrow \quad R_{n}=\Phi_{w}\left(G_{q}\right),
$$

for some $G_{q}$ and some $w=w_{1} w_{2} \cdots w_{m}$. In Sect. 5, we discuss the role of the generators and introduce the function $\gamma_{G}$ which gives the inner tube formula for a generator in the sense of (1.1). Moreover, appropriately parameterizing $\gamma_{G}$ yields the inner tube formula for a scaled generator. Therefore, by integrating $\gamma_{G}$ against $\eta_{\mathfrak{s}}$, one obtains the total contribution of $G_{q}$ (and its images under the maps $\Phi_{w}$ ) to the final tube formula $V_{\mathcal{T}}$. This is elaborated upon in Sect. 5.3.

At last, the tubular zeta function of the tiling $\zeta_{\mathcal{T}}$ is assembled from the scaling zeta function, the tiling, and the terms appearing in $\gamma_{G}$. In some precise sense, $\zeta_{\mathcal{T}}$ is a generating function for the geometry of the self-similar tiling. Using $\zeta_{\mathcal{T}}$, and following the distributional 
techniques and explicit formulas of [30], we are able to obtain an explicit distributional tube formula for self-similar tilings.

Outline The rest of this paper is organized as follows. Section 2 contains a quick overview of the background material concerning self-similar tilings. Section 3 discusses how the notion of inradius describes the different scales of the tiling. Section 4 defines the scaling measure, the scaling zeta function, and complex dimensions of a self-similar tiling. Section 5 develops the tube formula for the generators of a self-similar tiling, and establishes the general form of $V_{\mathcal{T}}(\varepsilon)$ in terms of this. Section 6 reviews the explicit formulas for fractal strings which will be used in the proof of the main results. Section 7 defines the tubular zeta function of the tiling, and states and proves the tube formula for fractal sprays (a generalization of a tiling) given in Theorem 7.4, from which the tube formula for self-similar tilings follows readily, and Section 9 discusses several examples illustrating the theory. Appendix A verifies the validity of the definition of the tubular zeta function $\zeta_{\mathcal{T}}$. Finally, Appendix B verifies the distributional error term and its estimate, from Theorem 7.4.

Remark 1.4 (A Note on the References) The primary references for this paper are [32] and the research monograph "Fractal Geometry, Complex Dimensions and Zeta Functions: Geometry and spectra of fractal strings" by Lapidus and van Frankenhuijsen [30]. This volume is essentially a revised and much expanded version of [27], by the same authors. The present paper cites [30] almost exclusively, so we provide the following partial correspondence between chapters for the aid of the reader:

\begin{tabular}{l|l|l|l|l|l|} 
[27] & Chap. 2 & Chap. 3 & Chap. 4 & Chap. 6 & Chap. 10 \\
\hline [30] & Chap. 2-3 & Chap. 4 & Chap. 5 & Chap. 8 & Chap. 12
\end{tabular}

Remark 1.5 Throughout, we reserve the symbol $\stackrel{\circ}{=} \sqrt{-1}$ for the imaginary number.

\section{The Self-Similar Tiling}

This section provides an overview of the necessary background material concerning selfsimilar tilings. Further details may be found in [32].

Definition 2.1 A self-similar system is a family $\left\{\Phi_{j}\right\}_{j=1}^{J}$ (with $J \geq 2$ ) of contraction similitudes

$$
\Phi_{j}(x):=r_{j} M_{j} x+a_{j}, \quad j=1, \ldots, J .
$$

For $j=1, \ldots, J$, we have $0<r_{j}<1, a_{j} \in \mathbb{R}^{d}$, and $M_{j} \in O(d)$, the orthogonal group of rigid rotations in $d$-dimensional Euclidean space $\mathbb{R}^{d}$. The number $r_{j}$ is the scaling ratio of $\Phi_{j}$. For convenience, assume that

$$
1>r_{1} \geq r_{2} \geq \cdots \geq r_{J}>0
$$

It is well known that there is a unique nonempty compact subset $F \subseteq \mathbb{R}^{d}$ satisfying the fixed-point equation

$$
F=\Phi(F):=\bigcup_{j=1}^{J} \Phi_{j}(F)
$$


This (self-similar) set $F$ is called the attractor of $\Phi$. We abuse notation and let $\Phi$ denote both an operator on compacta (as in (2.2)) and the family $\left\{\Phi_{j}\right\}$. Different self-similar systems may give rise to the same self-similar set; therefore we emphasize the self-similar system and its corresponding dynamics.

It is shown in [32] that for a self-similar system satisfying the tileset condition (see Definition 2.2), there exists a natural decomposition of $C \backslash F$ which is produced by the system $\Phi$. The construction of this tiling is illustrated for a well-known example, the Koch curve, in Fig. 1. It may help the reader to look at this example before diving into the next paragraph and the thicket of definitions therein. Further examples are depicted in Sect. 9.

Let $C:=[F]$ be the convex hull of $F$, and let $T:=$ relint $C$ be the relative interior of $C$. Iterates of the hull $C$ under $\Phi$ are denoted

$$
C_{k}:=\Phi^{k}(C)=\bigcup_{w \in \mathcal{W}_{k}} \Phi_{w}(C),
$$

where $w=w_{1} \ldots w_{k}$ is a word in $\mathcal{W}_{k}:=\{1,2, \ldots, J\}^{k}$ and $\Phi_{w}:=\Phi_{w_{k}} \circ \ldots \circ \Phi_{w_{2}} \circ \Phi_{w_{1}}$. For future reference, let $\mathcal{W}:=\bigcup_{k=1}^{\infty} \mathcal{W}_{k}$ be the set of all finite words $w$ over the alphabet $\{1,2, \ldots, J\}$.
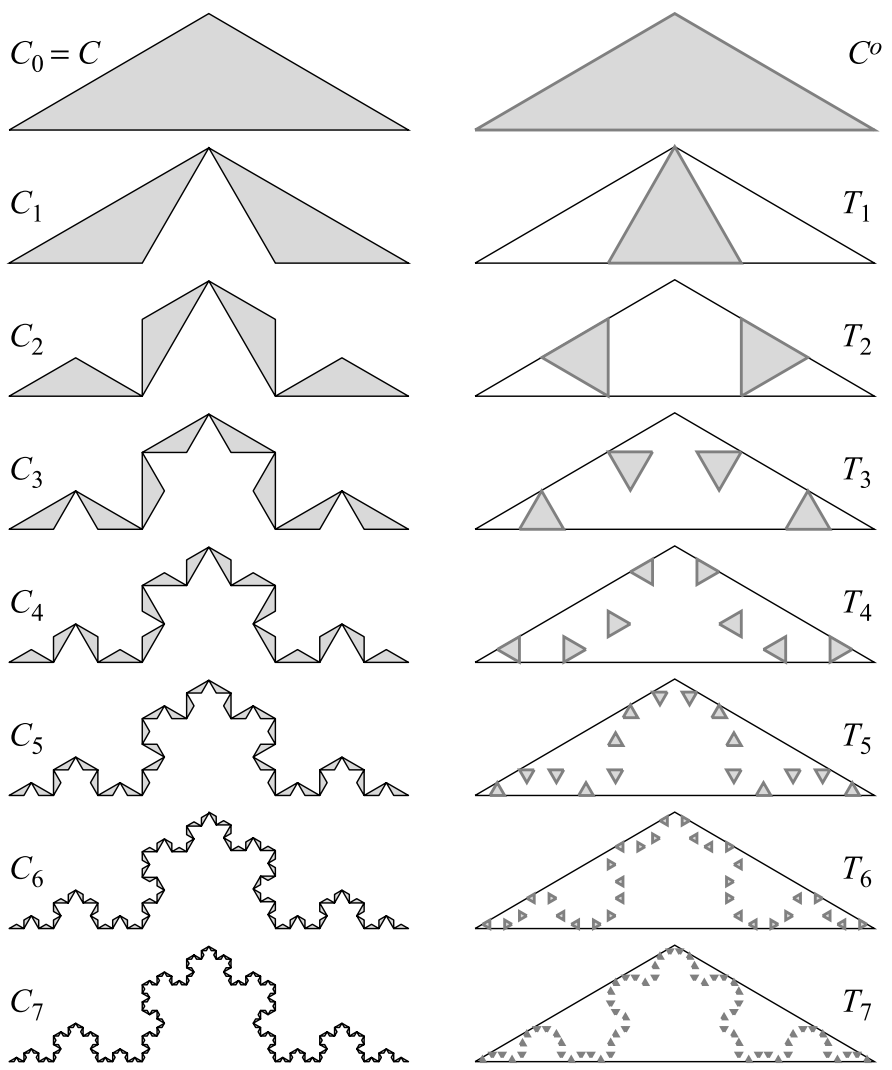

Fig. 1 Construction of the Koch tiling $\mathcal{K}$. This example is discussed further in Sect. 9. The tiling $\mathcal{K}$ has the single generator $T_{1}=G_{1}$, an equilateral triangle 
Definition 2.2 The system satisfies the tileset condition iff $T \nsubseteq \Phi(C)$ and

$$
\operatorname{int} \Phi_{j}(C) \cap \operatorname{int} \Phi_{\ell}(C)=\varnothing, \quad j \neq \ell
$$

This is a restriction on the overlap of the images of the mappings and implies (but is not equivalent to) the open set condition. For any system satisfying the tileset condition,

$$
T_{1}:=T \backslash C_{1}
$$

is well-defined and nonempty, and hence so is $T_{k}:=\Phi^{k}\left(T_{1}\right)$. As an open set, $T_{1}$ is a disjoint union of connected open sets:

$$
T_{1}=G_{1} \cup G_{2} \cup \cdots \cup G_{Q}, \quad G_{p} \cap G_{q}=\varnothing, p \neq q .
$$

Definition 2.3 The generators of the tiling are the connected components of $T_{1}$, i.e., the disjoint open sets $\left\{G_{q}\right\}$ in (2.6).

The number $Q$ of generators depends on the system $\Phi$, not just on $F$. In general, the number of connected components of an open subset of $\mathbb{R}^{d}$ may be countable; however, in this paper we assume $Q<\infty$.

Definition 2.4 The self-similar tiling of $\Phi$ is

$$
\mathcal{T}=\left\{R_{n}\right\}_{n=1}^{\infty}=\left\{\Phi_{w}\left(G_{q}\right) \vdots w \in \mathcal{W}, q=1, \ldots, Q\right\}
$$

In (2.7), the sequence $\left\{R_{n}\right\}$ is an enumeration of the sets $\left\{\Phi_{w}\left(G_{q}\right)\right\}$, and $\Phi_{w}$ is as in (2.3). We say $\mathcal{T}$ is a tiling of $C \backslash F$ because the tiles $R_{n}$ have disjoint interiors and $F$ does not intersect the interior of any $R_{n}$ (see Fig. 3):

$$
C=\bigcup_{n} \overline{R_{n}}, \quad F \subseteq \bigcup_{n} \partial R_{n}, \quad \text { and } \quad R_{n_{1}} \cap R_{n_{2}}=\partial R_{n_{1}} \cap \partial R_{n_{2}} .
$$

\section{The Inradius}

As alluded to in (1.1), we are interested in that portion of a set which lies within $\varepsilon$ of its boundary.

Definition 3.1 Given $\varepsilon>0$, the inner $\varepsilon$-neighborhood of a bounded set $A \subseteq \mathbb{R}^{d}, d \geq 1$, is

$$
A_{\varepsilon}:=\{x \in A \vdots \operatorname{dist}(x, \partial A) \leq \varepsilon\}
$$

where $\partial A$ is the boundary of $A$. We are primarily interested in the $d$-dimensional Lebesgue measure of $A_{\varepsilon}$, denoted $V_{A}(\varepsilon):=\operatorname{vol}_{d}\left(A_{\varepsilon}\right)$.

Remark 3.2 The primary reason we have worked with the inner $\varepsilon$-neighborhood instead of the exterior (as in (1.3)) is that it is more intrinsic to the set; it makes the computation independent of the embedding of $\mathcal{T}$ into $\mathbb{R}^{d}$. At least, this should be the case, provided the 'curvature' terms $\kappa_{k}$ of Definition 5.2 are also intrinsic. As a practical bonus, working with the inner $\varepsilon$-neighborhood allows us to avoid potential issues with the intersections of the $\varepsilon$-neighborhoods of different components. 
It is clear that if $A$ is a bounded set, $A \subseteq A_{\varepsilon}$ for sufficiently large $\varepsilon$. Alternatively, it is apparent that for a fixed $\varepsilon>0$, any sufficiently small set will be entirely contained within its $\varepsilon$-neighborhood. The notion of inradius allows us to see when this phenomenon occurs.

Definition 3.3 The inradius $\rho$ of a set $A$ is

$$
\rho=\rho(A):=\sup \{\varepsilon>0: \exists x \text { with } B(x, \varepsilon) \subseteq A\} .
$$

Note that the supremum is taken over $\varepsilon>0$, because $A_{0}=\bar{A}$. The inradii $\rho_{n}=\rho\left(R_{n}\right)$ replace the lengths $\ell_{n}=2 \rho\left(L_{n}\right)$ of the 1-dimensional theory; furthermore, the inradius is characterized by the following theorem. ${ }^{3}$

Theorem 3.4 In $\mathbb{R}^{d}$, the inradius is the furthest distance from a point of $A$ to $\partial A$, or the radius of the largest ball contained in A, i.e.,

$$
\rho(A)=\sup \left\{\varepsilon>0: V\left(A_{\varepsilon}\right)<V(A)\right\} .
$$

Proof The continuity of the distance and volume functionals gives $V\left(A_{\delta}\right)<V(A)$ if and only if there is a set $U$ of positive $d$-dimensional measure contained in the interior of $A$ which is further than $\delta$ from any point of $\partial A$. For any $x \in U, B(x, \delta) \subseteq A$. Conversely, for $\delta$ strictly less than the right-hand side of (3.3), the same reasons imply the existence of the set $U$ of positive measure.

Remark 3.5 The proof of Theorem 3.4 shows that the inradius may also be defined by $\rho(A)=\sup \{\operatorname{dist}(x, \partial A): x \in A\}$.

The utility of the inradius in the present paper arises primarily from the equality (3.3) and the fact that the inradius behaves well under the action of the self-similar system:

$$
\rho_{n}=\rho\left(R_{n}\right)=\rho\left(\Phi_{w}\left(G_{q}\right)\right)=r_{1}^{e_{1}} \cdots r_{J}^{e_{J}} g_{q}
$$

where $r_{j}$ is the scaling ratio of $\Phi_{j}$, and the exponent $e_{j} \in \mathbb{N}$ indicates the multiplicity of the letter $j$ in the finite word $w \in \mathcal{W}$.

Definition 3.6 For $q=1, \ldots, Q$, the $q^{\text {th }}$ generating inradius is the inradius of the $q^{\text {th }}$ generator of the tiling $\mathcal{T}$ and denoted

$$
g_{q}:=\rho\left(G_{q}\right)
$$

For convenience, we may take the generators in nonincreasing order, i.e., index the generators so that

$$
g_{1} \geq g_{2} \geq \cdots \geq g_{Q}>0
$$

\section{Measures and Zeta Functions}

In this section and the rest of the paper, any zeta function is understood to be the meromorphic extension of its defining expression.

\footnotetext{
${ }^{3}$ Theorem 3.4 is folkloric, but we were unable to find it in the literature and so have provided a proof.
} 


\subsection{The Geometric Zeta Function of a Fractal String}

We recall the notion of "fractal string" and "fractal spray from [30], see also [10-12, 18-20, 25-29, 33], along with [17, Example 5.1 and Appendix C].

Definition 4.1 A fractal string is defined to be a bounded open subset of $\mathbb{R}$, that is, a countable collection of disjoint open intervals, $L=\bigcup_{n=1}^{\infty} L_{n}$, with lengths $\mathcal{L}=\left\{\ell_{n}\right\}_{n=1}^{\infty}$. The geometric zeta function of such an object is

$$
\zeta_{\mathcal{L}}(s)=\sum_{n=1}^{\infty} \ell_{n}^{s},
$$

and can be used to study the geometry of $\mathcal{L}$ and of its (presumably fractal) boundary $\partial \mathcal{L}:=$ $\partial L$.

Observe that $\zeta_{\mathcal{L}}(s)$ is the Mellin transform of the measure

$$
\eta_{\mathcal{L}}=\sum_{n=1}^{\infty} \delta_{1 / \ell_{n}},
$$

where $\delta_{x}$ denotes the Dirac mass (or Dirac measure) at $x$. Thus,

$$
\zeta_{\mathcal{L}}(s)=\int_{0}^{\infty} x^{s} d \eta_{\mathcal{L}}(x) .
$$

The following definition first appeared in [26].

Definition 4.2 Let $G \subseteq \mathbb{R}^{d}$ be a nonempty bounded open set, which we will call the generator (or basic shape). Then a fractal spray $\mathcal{T}$ is a bounded open subset of $\mathbb{R}^{d}$ which is the disjoint union of open sets $\omega_{n}$ for $n=1,2, \ldots$, where each $\omega_{n}$ is congruent to $\ell_{n} G$, the homothetic of $G$ by $\ell_{n}$. Here, $\mathcal{L}=\left\{\ell_{n}\right\}_{n=1}^{\infty}$ is a fractal string and we say that $\mathcal{T}$ is "scaled by" $\mathcal{L}$. It is clear that a self-similar tiling as discussed in Definition 2.4 is a special case of fractal spray.

Thus, any fractal string can be thought of as a fractal spray on the generator $G=(0,1)$, the unit interval. In the context of the current paper, a self-similar tiling is a union of fractal sprays on the generators $G_{1}, \ldots, G_{Q}$, each scaled by a fixed self-similar string. A general fractal spray may have multiple generators, as long as they are all scaled by the same fractal string $\mathcal{L}$. However, for the remainder of this paper we consider only a single generator $G$. Indeed, as mentioned in Sect. 5.4, the multiple-generator case can readily be reduced to the case of a single generator.

Remark 4.3 One can also define a generalized fractal string $\eta$ to be a locally finite regular Borel measure on $\mathbb{R}_{+}$with support bounded away from 0 , as is done in [30, Sect. 4]. With regard to scaling, this is perhaps the greatest degree of generality in which the results of the present paper also hold. Indeed, all the main results of this paper remain true when $\eta_{\mathfrak{s}}$ is replaced by a generalized fractal string $\eta$, including the technical content of the appendices. However, since the geometric realization of a generalized fractal string (or spray) is unclear at this point, we describe tube formulas and other concepts from geometric measure theory in terms of $\eta_{\mathfrak{s}}$. 


\subsection{The Scaling Zeta Function}

We now extend Definition 4.1 to higher dimensions. In 1 dimension, the length of an interval is just twice its inradius, and the distinction between the scale of a set and its volume is blurred. In higher dimensions, the two are related by a power law, i.e., in $\mathbb{R}^{d}$, the volume of a compact set of full dimension will change by a factor of $r^{d}$ when the set is scaled by a factor $r$. In [30], the geometric zeta function $\zeta_{\mathcal{L}}$ records the measures of all the open sets comprising the string. However, in higher dimensions it is easier to use a zeta function $\left(\zeta_{\mathfrak{s}}\right.$, introduced just below) to record the scales (inradii) of the open sets in question.

In Definition 7.1, we will introduce the tubular zeta function of the tiling $\zeta_{\mathcal{T}}$, which is the higher-dimensional analogue of $\zeta_{\mathcal{L}}$, and is defined in terms of $\zeta_{\mathfrak{F}}$ and the generators. The tiling zeta function $\zeta_{\mathcal{T}}$ encodes the density of geometric states of $\mathcal{T}$ and acts as a generating function for the geometry of the entire tiling. The scaling zeta function encodes only scaling data (inradii). In [30], both of these roles are essentially played by $\zeta_{\mathcal{L}}$.

Definition 4.4 For a fractal spray scaled by a given fractal string $\mathcal{L}=\left\{\ell_{n}\right\}_{n=1}^{\infty}$, we use the inradii $\rho_{n}:=\frac{\ell_{n}}{2}$ to define the scaling measure by

$$
\eta_{\mathfrak{s}}(x):=\sum_{n=1}^{\infty} \delta_{1 / \rho_{n}}(x) .
$$

Definition 4.5 The scaling zeta function is the Mellin transform of the scaling measure:

$$
\zeta_{\mathfrak{s}}(s):=\int_{0}^{\infty} x^{-s} d \eta_{\mathfrak{s}}(x)
$$

Remark 4.6 In the special case of a self-similar tiling, as in Sect. 2, it is easy to see from Definition 4.2 that the scaling measure encodes the scaling factors of the self-similar system $\Phi$ as a sum of Dirac masses:

$$
\eta_{\mathfrak{s}}(x):=\sum_{w \in \mathcal{W}} \delta_{1 / r_{w}}(x)
$$

In this case, one can see that the scaling zeta function $\zeta_{\mathfrak{s}}$ encodes the combinatorics of the scaling ratios $\left\{r_{j}\right\}_{j=1}^{J}$ of $\Phi=\{\Phi\}_{j=1}^{J}$, and is thus a generating function for the scaling properties of $\Phi$ :

$$
\zeta_{\mathfrak{s}}(s)=\sum_{w \in \mathcal{W}} r_{w}^{s}=\sum_{k=0}^{\infty} \sum_{w \in \mathcal{W}_{k}} r_{w}^{s} .
$$

However, there is another, simpler (and more useful) form of $\zeta_{\mathfrak{s}}$, which we now explain.

Theorem 4.7 is the higher-dimensional counterpart of [30, Theorem 2.4], and can, in fact, be viewed as a corollary of it; see Sect. 4.3. Indeed, it is proved in precisely the same way.

Theorem 4.7 The scaling zeta function of a self-similar tiling is

$$
\zeta_{\mathfrak{s}}(s)=\frac{1}{1-\sum_{j=1}^{J} r_{j}^{s}} .
$$

This remains valid for the meromorphic extension of $\zeta_{\mathfrak{s}}$ to all of $\mathbb{C}$. 
Definition 4.8 We can now define the scaling (complex) dimensions of a tiling $\mathcal{T}$ as the poles of the scaling zeta function:

$$
\mathcal{D}_{\mathfrak{s}}:=\left\{\omega \in \mathbb{C}: \zeta_{\mathfrak{s}}(s) \text { has a pole at } \omega\right\}
$$

\subsection{Comparison with [30]}

Although the measure and zeta function introduced in Definition 4.4 and Definition 4.5 above correspond to fractal subsets of $\mathbb{R}^{d}$, it is crucial to note that (setting geometric interpretation aside) they are formally identical to the objects $\mathcal{L}$ (thought of as a measure) and $\zeta_{\mathcal{L}}$ studied in [30]. To be precise, the scaling measure $\eta_{\mathfrak{s}}$ is a fractal string of the sort studied in Chap. 2-3 of [30], and a generalized fractal string of the kind introduced in Chap. 4 of [30]. Consequently, all of the explicit formulas developed in [30] are applicable to the measures and zeta functions described in the present paper. This is key to the proof of Theorem 7.4, the tube formula for fractal sprays.

In the special case when $\eta_{\mathfrak{s}}$ is the scaling measure of a self-similar tiling, $\zeta_{\mathfrak{s}}$ may be thought of as the geometric zeta function of a self-similar string (as in [30]) with scaling ratios $\left\{r_{j}\right\}_{j=1}^{J}$ and a single gap, ${ }^{4}$ which has been normalized so as to have $\ell_{1}=1$, where $\ell_{1}$ is the first length in the string. Let $D$ be the unique real number satisfying $\sum_{j=1}^{J} r_{j}^{D}=1$. One can check (as in [30], Sect. 5.1) that for some real constant $c>D$,

$$
\eta_{\mathfrak{s}}(x)=\frac{1}{2 \pi \AA} \int_{c-i \infty}^{c+i \infty} x^{s-1} \zeta_{\mathfrak{s}}(s) d s, \text { and } \quad \zeta_{\mathfrak{s}}(s)=\int_{0}^{\infty} x^{-s} \eta_{\mathfrak{s}}(d x) .
$$

Additionally, the structure theorem for complex dimensions of self-similar strings [30, Theorem 3.6] (in the special case of a single gap), holds for the set of scaling complex dimensions of Definition 4.8. By (4.8), $\mathcal{D}_{\mathfrak{s}}$ consists of the set of complex solutions of the complexified Moran equation $\sum_{j=1}^{J} r_{j}^{s}=1$ which is studied in detail in [30, Chaps. 2-3]. In particular, the scaling complex dimensions lie in a horizontally bounded strip of the form $D_{\ell} \leq \operatorname{Re} s \leq D$, where $D$ is as just above and $D_{\ell}<D$ is some other (finite, possibly negative) constant. The positive number $D$ is called the similarity dimension of $\Phi$ (or of its attractor $F$ ) and coincides with the abscissa of convergence of $\zeta_{\mathfrak{s}}$ [30], Theorem 1.10. ${ }^{5}$ Furthermore, the following dichotomy prevails:

- Lattice case. When the logarithms of the scaling ratios $r_{j}$ are each an integer power of some common positive real number, the scaling complex dimensions lie periodically on finitely many vertical lines, including the line $\operatorname{Re} s=D$. In this case, there are infinitely many complex dimensions with real part $D$

- Nonlattice case. Otherwise, the scaling complex dimensions are quasiperiodically distributed and $s=D$ is the only complex dimension with real part $D$. However, there exists an infinite sequence of scaling complex dimensions approaching the line $\operatorname{Re} s=D$ from the left. In this case, the set $\{\operatorname{Re} s: s \in \mathcal{D}\}$ appears to be dense in (finitely many compact subintervals of) $\left[D_{\ell}, D\right]$.

\footnotetext{
${ }^{4}$ In this paper, we use the term "generator" in place of "gap".

If the self-similar system defining $F$ satisfies the 'open set condition' (see [14], as described in [2, 31, 39] or [15]), as when the tileset condition is satisfied, then $D$ coincides with the Hausdorff and Minkowski dimensions of $F$.
} 
It has been proven in [30] that for $d=1$, the attractor of $\Phi$ fails to be Minkowski measurable if and only if $\zeta_{\mathcal{L}}$ has nonreal complex dimensions with real part $D$, and in [30], Conj. 12.18, this is conjectured to hold also in higher dimensions. See also Remark 8.6.

\section{The Generators}

The inner tube formula for a self-similar tiling will consist of the sum of the inner tube formulas for each tile, and each of these can be expressed as a rescaled version of the tube formula for a generator. That is, if $R=\Phi_{w}(G)$ for some $w \in \mathcal{W}$, then the inradius of such a tile is

$$
\rho=\rho(R)=\rho\left(\Phi_{w}(G)\right)=r_{w} g=r_{1}^{e_{1}} \ldots r_{J}^{e_{J}} g
$$

and invariance of Lebesgue measure under rigid motions gives

$$
V_{R}(\varepsilon)=V_{\Phi_{w}(G)}(\varepsilon)=V_{r_{w} G}(\varepsilon) .
$$

Thus, it behooves us to find an expression for

$$
\gamma_{G}(x, \varepsilon):=V_{(1 / x) G}(\varepsilon)
$$

where $\frac{1}{x} G$ is a homothetic image of $G$, scaled by some factor $\frac{1}{x}>0$. Then $\gamma_{G}(1, \varepsilon)$ gives the inner tube formula for $G$, and $\gamma_{G}(x, \varepsilon)$ is the volume of a tile which is similar to $G$ but which has been scaled by $1 / x$. The motivation for defining $\gamma_{G}$ in terms of $1 / x$ (rather than $x$ ) appears in (5.17). Since $\gamma_{G}(x, \varepsilon)$ gives the inner tube formula for and set congruent to $(1 / x) G$, it will also be useful for computing the inner tube formula for a general fractal spray.

Definition 5.1 A Steiner-like representation ${ }^{6}$ of a generator $G$ is an inner tube formula of the form

$$
V_{G}(\varepsilon)=\sum_{k=0}^{d-1} \kappa_{k}(G, \varepsilon) \varepsilon^{d-k}, \quad \text { for } \varepsilon<g,
$$

where each $\kappa_{k}(G, \varepsilon)$ is some reasonably nice (e.g., bounded and locally integrable) function for $\varepsilon \in[0, \infty)$. In particular, we require that

(1) each $\kappa_{k}(G, \varepsilon)$ is homogeneous of degree $k$, so that for $\lambda>0$,

$$
\kappa_{k}(\lambda G, \lambda \varepsilon)=\kappa_{k}(G, \varepsilon) \lambda^{k}, \quad \text { and }
$$

(2) each $\kappa_{k}(G, \varepsilon)$ is rigid motion invariant, so that

$$
\kappa_{k}(T(G), \varepsilon)=\kappa_{k}(G, \varepsilon),
$$

for any (affine) isometry $T$ of $\mathbb{R}^{d}$.

\footnotetext{
${ }^{6}$ We are grateful to Steffen Winter for pointing out that (5.3) is really a property of (a choice of) the tube formula, as opposed to a property of the generator, and for suggesting the term "Steiner-like representation" (to replace the term "Steiner-like generator", which appeared in a previous draft).
} 


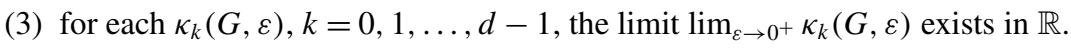

We have chosen the term "Steiner-like" for Definition 5.1 because the intrinsic volumes $\mu_{k}$ (see Theorem 1.1 and the ensuing discussion) satisfy the following properties:

(1) each $\mu_{k}$ is homogeneous of degree $k$, so that for $x>0$,

$$
\mu_{k}(x A)=\mu_{k}(A) x^{k}, \quad \text { and }
$$

(2) each $\mu_{k}(A)$ is rigid motion invariant, so that

$$
\mu_{k}(T(A))=\mu_{k}(A),
$$

for any (affine) isometry $T$ of $\mathbb{R}^{d}$.

Caution: the description of $\kappa_{k}(G)$ given in the conditions of Definition 5.1 is intended to emphasize the resemblance between $\kappa_{k}(G)$ and $\mu_{k}$. However, $\kappa_{k}(G)$ may be signed (even when $G$ is convex and $k=d-1, d)$ and is more complicated than $\mu_{k}$ in general. In contrast, the Federer curvature measures $\Theta_{k}$ are always positive for convex sets; cf. [4] and [37].

Note added in proof Property (3) of Definition 5.1 eventually proved to be unnecessary, and will be omitted in future work, e.g., [24].

\subsection{The Tube Formula for a Monophase Generator}

In this paper, we discuss only the special case of a Steiner-like generator $G$ where the coefficient functions $\kappa_{k}(G, \varepsilon)$ are piecewise constant functions of $\varepsilon$, in which case $G$ is called pluriphase as in Definition 5.5. In the special case when each $\kappa_{k}(G, \varepsilon)$ takes on only two values, $G$ is called monophase as in Definition 5.2. We will treat the general case in the forthcoming collaboration with Steffen Winter [24].

Definition 5.2 A Steiner-like generator $G$ is said to be a monophase generator, or to have a monophase tube formula, iff

$$
V_{G}(\varepsilon)=\gamma_{G}(1, \varepsilon)=\sum_{k=0}^{d-1} \kappa_{k}(G) \varepsilon^{d-k}, \quad \text { for } \varepsilon<g,
$$

for some $\kappa_{k}(G) \in \mathbb{R}, k=0,1, \ldots, d-1$.

Not every polyhedral generator $G$ is monophase; the more general pluriphase case is discussed in Sect. 5.2. In general, the computation of $V(G, \varepsilon)$ may be nontrivial. So far, we have only defined $V(G, \varepsilon)$ for $\varepsilon<g$. To extend it to all of $\mathbb{R}^{+}$, note that $V(G, \varepsilon)$ is just the Lebesgue measure of $G$ for $\varepsilon \geq g$. Therefore, define

$$
\begin{aligned}
& \kappa_{k}(G, \varepsilon):=\kappa_{k}(G) \chi_{[0, g)}(\varepsilon), \quad k=0,1, \ldots, d-1, \\
& \kappa_{d}(G, \varepsilon):=-\kappa_{d}(G) \chi_{[g, \infty)}(\varepsilon),
\end{aligned}
$$

where $\kappa_{k}(G)$ is as in (5.8), $\chi_{A}$ is the usual characteristic function of the set $A$, and $\kappa_{d}(G)$ is defined to be the negative of the $d$-dimensional Lebesgue measure of $G$ :

$$
\kappa_{d}(G)=-\operatorname{vol}_{d}(G)
$$


Now we have

$$
V(G, \varepsilon)=\sum_{k=0}^{d} \kappa_{k}(G, \varepsilon) \varepsilon^{d-k}, \quad \text { for } \varepsilon \geq 0
$$

Remark 5.3 Note that for the case $k=d$, one must have $\lim _{\varepsilon \rightarrow 0^{+}} \kappa_{d}(G, \varepsilon)=0$. This implies that $V(G, \varepsilon)$ can have no constant term when $G$ is monophase, and hence the sum in (5.8) does not include a $d^{\text {th }}$ term. (The same may not hold when $G$ is pluriphase.)

Theorem 5.4 If $G$ is monophase, then for any tile congruent to the homothetic image $\frac{1}{x} G$, the inner tube formula is given by

$$
\gamma_{G}(x, \varepsilon)= \begin{cases}\sum_{k=0}^{d-1} \kappa_{k}(G) x^{-k} \varepsilon^{d-k}, & \varepsilon \leq g / x \\ -\kappa_{d}(G) x^{-d}, & \varepsilon \geq g / x\end{cases}
$$

Proof So far, we have only defined $V_{G}(\varepsilon)$ for $\varepsilon<g$. To extend it to all of $\mathbb{R}^{+}$, note that $V_{G}(\varepsilon)$ is just the Lebesgue measure of $G$ for $\varepsilon \geq g$. Therefore, define

$$
\begin{aligned}
\kappa_{k}(G ; \varepsilon) & :=\kappa_{k}(G) \chi_{[0, g)}(\varepsilon), \quad i=0,1, \ldots, d-1, \\
\kappa_{d}(G ; \varepsilon) & :=-\kappa_{d}(G) \chi_{[g, \infty)}(\varepsilon),
\end{aligned}
$$

where $\kappa_{k}(G)$ is as in (5.8), $\chi_{A}$ is the usual characteristic function of the set $A$, and $\mu_{d}$ is Lebesgue measure on $\mathbb{R}^{d}$. Now we have

$$
V_{G}(\varepsilon)=\gamma_{G}(1, \varepsilon)=\sum_{k=0}^{d} \kappa_{k}(G ; \varepsilon) \varepsilon^{d-k}, \quad \text { for } \varepsilon \geq 0 .
$$

Next, we would like to adapt this formula so as to obtain a tube formula valid for a tile of any size. Note that $V_{r G}(r \varepsilon)=r^{d} V_{G}(\varepsilon)$, as both expressions are measuring congruent regions in $\mathbb{R}^{d}$. Hence for $\varepsilon<g$, one has

$$
\sum_{k=0}^{d-1} r^{k} \kappa_{k}(G ; \varepsilon)(r \varepsilon)^{d-k}=r^{d} V_{G}(\varepsilon)=V_{r G}(r \varepsilon)=\sum_{k=0}^{d-1} \kappa_{k}(r G ; r \varepsilon)(r \varepsilon)^{d-k},
$$

and thus for $\varepsilon<g / x$, one has

$$
\gamma_{G}(x, \varepsilon)=V_{\frac{1}{x} G}(\varepsilon)=\sum_{k=0}^{d-1}\left(\frac{1}{x}\right)^{k} \kappa_{k}(G ; x \varepsilon) \varepsilon^{d-k}=\sum_{k=0}^{d-1} \kappa_{k}(G ; x \varepsilon) x^{-k} \varepsilon^{d-k} .
$$

Since $\kappa_{k}(G ; x \varepsilon)=\kappa_{k}(G) \chi_{[0, g)}(x \varepsilon)=\kappa_{k}(G) \chi_{[0, g / x)}(\varepsilon)$ for $k=0,1, \ldots, d-1$, it is clear that (5.2) may be expressed as

$$
\gamma_{G}(x, \varepsilon)=\sum_{k=0}^{d} \kappa_{k}\left(\frac{1}{x} G ; \varepsilon\right) \varepsilon^{d-k}= \begin{cases}\sum_{k=0}^{d-1} \kappa_{k}(G) x^{-k} \varepsilon^{d-k}, & \varepsilon \leq g / x, \\ -\kappa_{d}(G) x^{-d}, & \varepsilon \geq g / x,\end{cases}
$$

where the constants $\kappa_{k}(G)$ are as defined in (5.8) for $k=0,1, \ldots, d-1$, and in (5.9) for $k=d$. 
The function $\gamma_{G}(x, \varepsilon)$ gives the volume of the $\varepsilon$-neighborhood of a tile which is congruent to a generator scaled by $1 / x$. The value $\varepsilon=g / x$ corresponds to the value of $\varepsilon$ at which the inner $\varepsilon$-neighborhood of the tile becomes equal to the tile itself. Thus, $\gamma_{G}$ is continuous (but generally not differentiable) at $\varepsilon=g / x$.

\subsection{More General Generators}

Not every generator $G$ is monophase, so we introduce the pluriphase case in Definition 5.5. The most general case is discussed in [24]. In fact, even if $G$ is polyhedral or convex, it still may not be monophase. Example 5.6 gives a set which is convex and pluriphase but not monophase and the Cantor Carpet discussed in [24, Ex. 6.1] gives a tiling with a nonconvex generator (in the shape of a Swiss cross) which is polyhedral but not even pluriphase.

Definition 5.5 A generator $G$ is said to be a pluriphase generator iff its inner tube formula is given by a piecewise polynomial function of $\varepsilon$. Equivalently, $G$ is pluriphase iff the functions $\kappa_{k}(G ; \varepsilon)$ of (5.3) are piecewise constant for $k=0,1, \ldots, d$.

It is possible (though doubtful) that all convex generators are pluriphase, but this has not yet been proved. However, it seems likely that all convex polyhedra are pluriphase. For situations even more general, it is an interior version of Federer's notion of reach (see [4]) that is required. For such cases, the inner tube formula should be obtained in [23] via the more general methods of [13] and others, just like Theorem 5.4.

Example 5.6 (A Pluriphase Generator) Consider a fractal spray on a generator $G$ consisting of a $2 \times 2$ square with one corner replaced by a circular arc, as depicted in Fig. 2. This generator has inradius $g=\rho(G)=1$ and is pluriphase, but not monophase. Indeed, the relevant partition is

$$
\left\{0=\varepsilon_{0}, \varepsilon_{1}=1 / 2, \varepsilon_{2}=1\right\},
$$

and the tube formula for $G$ is

$$
\gamma_{G}(1, \varepsilon)= \begin{cases}\left(8+\frac{\pi}{4}\right) \varepsilon-\left(5+\frac{\pi}{4}\right) \varepsilon^{2}, & \varepsilon_{0} \leq \varepsilon \leq \varepsilon_{1}, \\ \frac{\pi-4}{16}+8 \varepsilon-4 \varepsilon^{2}, & \varepsilon_{1} \leq \varepsilon \leq \varepsilon_{2}, \\ \frac{\pi-4}{16}+4, & \varepsilon_{2} \leq \varepsilon\end{cases}
$$
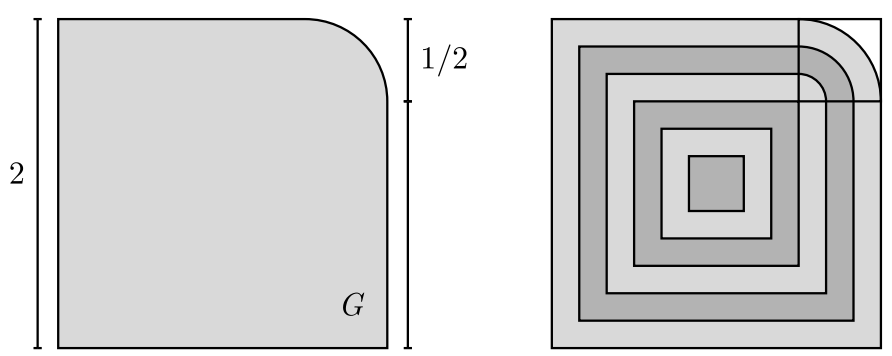

Fig. 2 A pluriphase generator which is not monophase 


\subsection{Tilings with One Generator}

Suppose we have a tiling $\mathcal{T}$ with just one generator $G$. Then the inner tube formula of $\mathcal{T}$ is given by

$$
V_{\mathcal{T}}(\varepsilon)=\sum_{n=1}^{\infty} V_{R_{n}}(\varepsilon)=\sum_{\rho_{n} \geq \varepsilon} V_{R_{n}}(\varepsilon)+\sum_{\rho_{n}<\varepsilon} V_{R_{n}}(\varepsilon),
$$

much as in [26], (3.2). Recall that $\rho_{n}$ is the inradius of the tile $R_{n}$. For $R_{n}=\Phi_{w}\left(G_{q}\right)$, invariance under rigid motions allows us to use the equality (5.1) to rewrite the sums in (5.16) as integrals with respect to the scaling measure $\eta_{\mathfrak{s}}$ :

$$
\begin{aligned}
V_{\mathcal{T}}(\varepsilon) & =\sum_{\rho_{n}^{-1} \leq 1 / \varepsilon} V_{R_{n}}(\varepsilon)+\sum_{\rho_{n}^{-1}>1 / \varepsilon} V_{R_{n}}(\varepsilon) \\
& =\int_{0}^{g / \varepsilon} V_{(1 / x) G}(\varepsilon) d \eta_{\mathfrak{s}}(x)+\mu_{d}(G) \int_{g / \varepsilon}^{\infty} x^{-d} d \eta_{\mathfrak{s}}(x) \\
& =\int_{0}^{\infty} \gamma_{G}(x, \varepsilon) d \eta_{\mathfrak{s}}(x) \\
& =\left\langle\eta_{\mathfrak{s}}, \gamma_{G}\right\rangle,
\end{aligned}
$$

where $\gamma_{G}$ is a 'test function' giving the volume of a tile which is similar to $G$, but which has been scaled by a factor of $1 / x$; see (5.2). Although $\gamma_{G}$ is not smooth, it fits the criteria given in Theorem 6.4 and is thus amenable to the distributional techniques of [30, Sect. 5.4].

\subsection{Tilings with Multiple Generators}

Upon replacing $G$ by $G_{q}$, we use the notation $V_{q}, \gamma_{q}, \kappa_{q k}$, etc., to refer to the corresponding quantity for the $q^{\text {th }}$ generator. For example, $\gamma_{G}(x, \varepsilon)$ is replaced by $\gamma_{q}(x, \varepsilon)=\gamma_{G_{q}}(x, \varepsilon)$, the volume of the $\varepsilon$-neighborhood of a tile which is similar to $G_{q}$ but which has been scaled by $x$.

The contribution to $V_{\mathcal{T}}(\varepsilon)$ resulting from one generator $G_{q}$ and its successive images is $V_{q}(\varepsilon):=\left\langle\eta_{\mathfrak{s}}, \gamma_{q}\right\rangle$, so the case of multiple generators can be reduced to a sum of singlegenerator tilings via the formula

$$
V_{\mathcal{T}}(\varepsilon)=\sum_{q=1}^{Q} V_{q}(\varepsilon)=\sum_{q=1}^{Q}\left\langle\eta_{\mathfrak{s}}, \gamma_{q}\right\rangle
$$

For a concrete example of how this is done, see the example of the pentagasket in Sect. 9.4.

Henceforth, we will always assume there is only a single generator, as this simplifying assumption will clarify the exposition. The single generator will always be denoted by $G$.

\section{Distributional Explicit Formulas for Fractal Strings}

These four definitions and the three theorems that follow them are adapted from [30, Sect. 5.3]. The technical details described here are used in the proof of Theorem 7.4, especially in Appendix A and Appendix B. The reader can easily skim or skip this section on a first reading. 
Definition 6.1 Let $f: \mathbb{R} \rightarrow \mathbb{R}$ be a bounded Lipschitz continuous function. Then the screen is $S=\left\{f(t)+{ }^{\circ} t: t \in \mathbb{R}\right\}$, the graph of a function with the axes interchanged. We let

$$
\begin{aligned}
\inf S & :=\inf _{t} f(t)=\inf \{\operatorname{Re} s: s \in S\}, \quad \text { and } \\
\sup S & :=\sup _{t} f(t)=\sup \{\operatorname{Re} s: s \in S\} .
\end{aligned}
$$

The screen is thus a vertical contour in $\mathbb{C}$. The region to the right of the screen is the set $W$, called the window:

$$
W:=\{z \in \mathbb{C}: \operatorname{Re} z \geq f(\operatorname{Im} z)\}
$$

The poles of $\zeta_{\mathfrak{s}}$ are called the scaling dimensions and those which lie in the window are called the visible scaling dimensions; the set of them is denoted

$$
\mathcal{D}_{\mathfrak{s}}(W)=\left\{\omega \in W: \lim _{s \rightarrow \omega}\left|\zeta_{\mathfrak{s}}(s)\right|=\infty\right\} .
$$

Definition 6.2 The scaling measure $\eta_{\mathfrak{s}}$ (as in Definition 4.4) is said to be languid if its associated zeta function $\zeta_{\mathfrak{s}}$ satisfies certain growth conditions relative to the screen. Specifically, let $\left\{T_{n}\right\}_{n \in \mathbb{Z}}$ be a sequence in $\mathbb{R}$ such that $T_{-n}<0<T_{n}$ for $n \geq 1$, and

$$
\lim _{n \rightarrow \infty} T_{n}=\infty, \quad \lim _{n \rightarrow \infty} T_{-n}=-\infty, \quad \text { and } \quad \lim _{n \rightarrow \infty} \frac{T_{n}}{\left|T_{-n}\right|}=1 .
$$

For $\eta_{\mathfrak{s}}$ to be languid, there must exist real constants $\varpi, c>0$ and a sequence $\left\{T_{n}\right\}$ as described in (6.5), such that

L1 For all $n \in \mathbb{Z}$ and all $\sigma \geq f\left(T_{n}\right)$,

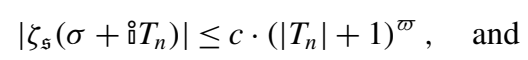

L2 For all $t \in \mathbb{R},|t| \geq 1$,

$$
\left|\zeta_{\mathfrak{s}}(f(t)+\stackrel{\circ}{t})\right| \leq c \cdot|t|^{\varpi} .
$$

In this case, $\eta_{\mathfrak{s}}$ is said to be languid of order $\varpi$.

Definition 6.3 The scaling measure $\eta_{\mathfrak{s}}$ is said to be strongly languid if it satisfies $\mathbf{L 1}$ and the condition $\mathbf{L} \mathbf{2}^{\prime}$, which is clearly stronger than $\mathbf{L 2}$ :

L2' There exists a sequence of screens $S_{m}(t)=f_{m}(t)+\stackrel{\circ}{ } t$ for $m \geq 1, t \in \mathbb{R}$, with $\sup S_{m} \rightarrow$ $-\infty$ as $m \rightarrow \infty$, and with a uniform Lipschitz bound. Additionally, there must exist constants $A, c>0$ such that

$$
\left|\zeta_{\mathfrak{s}}(f(t)+\stackrel{\circ}{\imath} t)\right| \leq c \cdot A^{\left|f_{m}(t)\right|}(|t|+1)^{\varpi},
$$

for all $t \in \mathbb{R}$ and $m \geq 1$.

It turns out that Definition 6.3 is always satisfied when $\eta_{\mathfrak{s}}$ is the scaling measure of a self-similar tiling; see [30, Sect. 6.4].

Taking [30, Theorem 5.26 and Theorem 5.30] at level $k=0$ gives the following distributional explicit formula for the action of a scaling measure $\eta_{\mathfrak{s}}$ on a test function $\psi \in C^{\infty}(0, \infty)$. Note that $\psi$ may not have compact support; only the decay properties (6.9)(6.10) are required. 
Theorem 6.4 (Extended Distributional Explicit Formula) Let $\eta_{\mathfrak{s}}$ be a scaling measure which is languid of order $\varpi$. Let $\psi \in C^{\infty}(0, \infty)$ with $n^{\text {th }}$ derivative satisfying, for some $\delta>0$, and every integer $n \in\{0,1, \ldots, N=[\varpi]+2\}$,

$$
\begin{gathered}
\psi^{(n)}(x)=O\left(x^{-n-D-\delta}\right) \quad \text { as } x \rightarrow \infty, \quad \text { and } \\
\psi^{(n)}(x)=\sum_{\alpha} a_{\alpha}^{(n)} x^{-\alpha-n}+O\left(x^{-n-\inf S+\delta}\right) \quad \text { as } x \rightarrow 0^{+} .
\end{gathered}
$$

Then we have the following distributional explicit formula for $\eta_{\mathfrak{s}}$ :

$$
\left\langle\eta_{\mathfrak{s}}, \psi\right\rangle=\sum_{\omega \in \mathcal{D}_{\mathfrak{s}}} \operatorname{res}\left(\zeta_{\mathfrak{s}}(s) \tilde{\psi}(s) ; \omega\right)+\sum_{\alpha \in W \backslash \mathcal{D}_{\mathfrak{s}}} a_{\alpha}^{(0)} \zeta_{\mathfrak{s}}(\alpha)+\langle\mathcal{R}, \psi\rangle,
$$

where the error term $\mathcal{R}(x)$ is the distribution given by

$$
\langle\mathcal{R}, \psi\rangle=\frac{1}{2 \pi \AA} \int_{S} \zeta_{\mathfrak{s}}(s) \tilde{\psi}(s) d s
$$

and estimated by

$$
\mathcal{R}(x)=O\left(x^{\sup S-1}\right), \quad \text { as } x \rightarrow \infty .
$$

Here, $\tilde{\psi}$ is the Mellin transform of the function $\psi$, defined by

$$
\tilde{\psi}(s):=\int_{0}^{\infty} x^{s-1} \psi(x) d x .
$$

Note: the sum in (6.10) is over finitely many complex exponents $\alpha$ with $\operatorname{Re} \alpha>-\sigma_{l}+\delta$; we express this by saying that $\psi$ has an asymptotic expansion of order $-\sigma_{l}+\delta$ at 0 .

Taking [30], Theorem 5.27, at level $k=0$ gives the following distributional explicit formula for the action of a scaling measure $\eta_{\mathfrak{s}}$ on a test function $\psi$. Note that in addition to requiring $\psi \in C^{\infty}(0, \infty)$, we now also require that $\psi$ is a finite linear combination of terms $x^{-\beta} e^{-c_{\beta} x}$ in a neighborhood of the interval $(0, A]$, where $A$ is the same constant as in Definition 6.3.

Theorem 6.5 (Extended Distributional Formula, without Error Term) Let $\eta_{\mathfrak{s}}$ be a strongly languid scaling measure. Let $q \in \mathbb{N}$ be such that $q>\max \{1, \varpi\}$, where $\varpi$ is as in Definition 6.2. Further, let $\psi$ be a test function that is $q$ times continuously differentiable on $(0, \infty)$. Assume that the $j^{\text {th }}$ derivative $\psi^{(j)}(x)$ satisfies (6.9) and (6.10), and that there exists a $\delta>0$ such that

$$
\psi^{(j)}(x)=\sum_{\alpha} a_{\alpha}^{(j)} x^{-\alpha} e^{-c_{\alpha} x}, \quad \text { for } x \in(0, A+\delta), 0 \leq j \leq q .
$$

Then formula (6.11) holds with $\mathcal{R} \equiv 0$.

Theorem 6.6 (Tube Formula for Fractal Strings [30], Theorem 8.1) Let $\mathcal{L}=\left\{\ell_{n}\right\}_{n=1}^{\infty}$ be a fractal string with languid zeta function $\zeta_{\mathcal{L}}=\sum_{n=1}^{\infty} \ell_{n}^{s}$. Then the volume of the (onesided) tubular neighborhood of radius $\varepsilon$ of the boundary of $\mathcal{L}$ is given by the following 
distributional explicit formula for test functions $\psi \in C_{c}^{\infty}(0, \infty)$, the space of $C^{\infty}$ functions with compact support contained in $(0, \infty)$ :

$$
V_{\mathcal{L}}(\varepsilon)=\sum_{\omega \in \mathcal{D}_{\mathcal{L}}(W)} \operatorname{res}\left(\frac{\zeta_{\mathcal{L}}(s)(2 \varepsilon)^{1-s}}{s(1-s)} ; \omega\right)+\left\{2 \varepsilon \zeta_{\mathcal{L}}(0)\right\}+\mathcal{R}(\varepsilon)
$$

Here the term in braces is only included if $0 \in W \backslash \mathcal{D}_{\mathfrak{s}}(W)$, and $\mathcal{R}(\varepsilon)$ is the error term, given by

$$
\mathcal{R}(\varepsilon)=\frac{1}{2 \pi \AA} \int_{S} \frac{\zeta_{\mathcal{L}}(s)(2 \varepsilon)^{1-s}}{s(1-s)} d s
$$

and estimated by

$$
\mathcal{R}(\varepsilon)=O\left(\varepsilon^{1-\sup S}\right), \quad \text { as } \varepsilon \rightarrow 0^{+}
$$

The meaning of (6.13) and (6.18), the order of the distributional error term, is given in Definition B.6 of Appendix B. When $\mathcal{L}$ is a self-similar fractal string, the results of Theorem 6.6 may be strengthened as described in Sect. 8.1 and in [30, Sect. 8.4]. In particular, one may take $W=\mathbb{C}$ and $\mathcal{R} \equiv 0$.

\section{The Tube Formula for Fractal Sprays}

We now present the main result of the paper, a higher-dimensional analogue of Theorem 6.6. While the proof is similar in spirit to the proof of the tube formula for fractal strings obtained in [30, Sect. 8.1] (cf. Theorem 6.6), it is significantly more involved, especially if Appendices $\mathrm{A}$ and $\mathrm{B}$ are taken into account. This result provides new insight, particularly with regard to the geometric interpretation of the terms of the formula; see Remark 10.2. Also, it introduces the proper conceptual framework and confirms that fractal sprays are clearly the higher-dimensional counterpart of fractal strings. In a similar vein, we will see from Theorem 8.3 (the tube formula for self-similar tilings) that the self-similar tilings are the natural higher-dimensional analogue of self-similar fractal strings.

Although our primary goal in this paper is to obtain a tube formula for self-similar tilings, we state our main result for the more general class of fractal sprays, as we expect it to be useful in the study of other fractal structures and tilings to be investigated in future work. The key special case of self-similar tilings is stated in Theorem 8.3 of Sect. 8.1.

\subsection{Statement of the Tube Formula}

We will prove the tube formula first for the more general case of fractal sprays, and then refine this result to obtain the formula for self-similar tilings.

Definition 7.1 Let $\mathcal{T}$ be a fractal spray with a single monophase generator $G$. Then the tubular zeta function (or volume zeta function) of $\mathcal{T}$ is

$$
\zeta_{\mathcal{T}}(\varepsilon, s):=\varepsilon^{d-s} \zeta_{\mathfrak{s}}(s) \sum_{k=0}^{d} \frac{g^{s-k}}{s-k} \kappa_{k}
$$

Here, $\kappa_{k}=\kappa_{k}(G)$ as defined in Definition 5.2. 
It turns out that $\zeta_{\mathcal{T}}$ is a meromorphic distribution-valued function for each fixed $s \in W$, where $W \subseteq \mathbb{C}$ is the window defined in Definition 6.1. This verification is given in Definition A.5 and Theorem A.7 of Appendix A. Considered as a distribution, the action of $\zeta_{\mathcal{T}}(s, \cdot)$ on a test function $\psi \in C_{c}^{\infty}(0, \infty)$ is given by

$$
\left\langle\zeta_{\mathcal{T}}(\varepsilon, s), \psi(\varepsilon)\right\rangle=\int_{0}^{\infty} \zeta_{\mathcal{T}}(\varepsilon, s) \psi(\varepsilon) d \varepsilon
$$

Here, $C_{c}^{\infty}(0, \infty)$ is the space of smooth functions with compact support contained in $(0, \infty)$. At first glance, it may appear strange that something as concretely geometric as a tube formula is given distributionaly. However, the flexibility of the distributional framework allows the proof to proceed; see [30, Remark 5.20].

Remark 7.2 The presentation here differs slightly from that given in [30], wherein the "geometric zeta function" is actually closer to what we call the scaling zeta function here. The general tube formula (6.16) involves the one-dimensional case of $\zeta_{\mathcal{T}}$, although this is not explicitly stated. For several reasons, it behooves one to think of $\zeta_{\mathcal{T}}$ as the zeta function most naturally associated with the geometric properties of the spray (or tiling), especially as pertains to the tube formula:

(i) The function $\zeta_{\mathcal{T}}$ arises naturally in the expression of the tube formula for the tiling, as will be seen in Theorem 7.4 and Theorem 8.3.

(ii) It is the poles of $\zeta_{\mathcal{T}}(\varepsilon, s)$ that naturally index the sum appearing in $V_{\mathcal{T}}$, and the residues of $\zeta_{\mathcal{T}}$ that give the actual volume.

(iii) Using $\zeta_{\mathcal{T}}$ leads to the natural unification of expressions which previously appeared unrelated; compare (8.2) to (8.4) in Corollary 8.7.

Thus, the function $\zeta_{\mathcal{T}}$ encodes all the geometric information of $\mathcal{T}$ as pertains to its tube formula. In Remark 8.13 we discuss how the unification mentioned in (ii) leads to a geometric interpretation of the term $\left\{2 \varepsilon \zeta_{\mathfrak{s}}(0)\right\}$ that appears in (6.16).

Definition 7.3 The set of complex dimensions of a fractal spray is

$$
\mathcal{D}_{\mathcal{T}}:=\mathcal{D}_{\mathfrak{s}} \cup\{0,1, \ldots, d-1\},
$$

where $\mathcal{D}_{\mathfrak{s}}$ is the set of poles of $\zeta_{\mathfrak{s}}$, as in (6.4). When a window $W$ has been specified, the set of visible complex dimensions is $\mathcal{D}_{\mathcal{T}}(W):=\mathcal{D}_{\mathcal{T}} \cap W$, and $\mathcal{D}_{\mathfrak{s}}(W)=\mathcal{D}_{\mathfrak{s}} \cap W$ is the set of visible scaling dimensions. Thus, $\mathcal{D}_{\mathcal{T}}(W)$ consists of the visible scaling dimensions and the visible "integral dimensions" of the spray. Furthermore, the poles of $\zeta_{\mathcal{T}}$ are all contained in $\mathcal{D}_{\mathcal{T}}$. Note that $\mathcal{D}_{\mathcal{T}}(W)$ is a discrete subset of $W \subseteq \mathbb{C}$, and hence is countable.

Theorem 7.4 (Tube Formula for Fractal Sprays) Let $\mathcal{T}$ be a fractal spray on the monophase generator $G$, with generating inradius $g=\rho(G)>0$, and scaling measure $\eta_{\mathfrak{s}}$. Assume that $\zeta_{\mathfrak{s}}$ is languid on a screen $S$ which avoids the dimensions in $\mathcal{D}_{\mathcal{T}}(W)$. Then for test functions in $C_{c}^{\infty}(0, \infty)$, the d-dimensional volume of the inner tubular neighborhood of the spray is given by the following distributional explicit formula:

$$
V_{\mathcal{T}}(\varepsilon)=\sum_{\omega \in \mathcal{D}_{\mathcal{T}}(W)} \operatorname{res}\left(\zeta_{\mathcal{T}}(\varepsilon, s) ; \omega\right)+\mathcal{R}(\varepsilon)
$$


where the sum ranges over the set (7.3) of visible integral and scaling dimensions of the spray. Here, the error term $\mathcal{R}(\varepsilon)$ is given by

$$
\mathcal{R}(\varepsilon)=\frac{1}{2 \pi \AA} \int_{S} \zeta_{\mathcal{T}}(\varepsilon, s) d s,
$$

and estimated by

$$
\mathcal{R}(\varepsilon)=O\left(\varepsilon^{d-\sup S}\right), \quad \text { as } \varepsilon \rightarrow 0^{+} .
$$

In the case that $\omega \in \mathcal{D}_{\mathfrak{s}}(W) \cap\{0,1, \ldots, d-1\}$, then the corresponding term res $\left(\zeta_{\mathcal{T}}(\varepsilon, s) ; \omega\right)$ appears only once in the sum in (7.4). As a distributional formula, (7.4) is valid when applied to test functions $\psi \in C_{c}^{\infty}(0, \infty)$. The order of the distributional error term as in (7.6) is defined in Definition B.6. There is a version of this theorem in which the error term vanishes identically; it is presented in Corollary 8.1. Also, the special case of self-similar tilings is presented in Theorem 8.3. The following proof relies heavily on the material in Sect. 6; the reader may wish to review this material before proceeding.

\subsection{Proof of the Tube Formula}

The reader may now wish to review Sect. 6 before proceeding, as the proof uses these explicit formulas and distributional techniques from [30].

Proof of Theorem 7.4 Recall that we view $V_{\mathcal{T}}(\varepsilon)$ as a distribution, ${ }^{7}$ so we understand $V_{\mathcal{T}}(\varepsilon)=\left\langle\eta_{\mathfrak{s}}, \gamma_{G}\right\rangle$ by computing its action on a test function $\psi$ :

$$
\begin{aligned}
\left\langle V_{\mathcal{T}}(\varepsilon), \psi\right\rangle=\left\langle\left\langle\eta_{\mathfrak{s}}, \gamma_{G}\right\rangle, \psi\right\rangle & =\int_{0}^{\infty}\left(\int_{0}^{\infty} \gamma_{G}(x, \varepsilon) d \eta_{\mathfrak{s}}(x)\right) \psi(\varepsilon) d \varepsilon \\
& =\int_{0}^{\infty} \int_{0}^{\infty} \gamma_{G}(x, \varepsilon) \psi(\varepsilon) d \varepsilon d \eta_{\mathfrak{s}}(x) \\
& =\left\langle\eta_{\mathfrak{s}},\left\langle\gamma_{G}, \psi\right\rangle\right\rangle .
\end{aligned}
$$

Now, writing $\kappa_{k}=\kappa_{k}(G)$, we use (5.11) to compute

$$
\begin{aligned}
\left\langle\gamma_{G}, \psi\right\rangle & =\int_{0}^{\infty} \gamma_{G}(x, \varepsilon) \psi(\varepsilon) d \varepsilon \\
& =\int_{0}^{\infty} \sum_{k=0}^{d} \kappa_{k}\left(\frac{1}{x} G ; \varepsilon\right) \varepsilon^{d-k} \psi(\varepsilon) d \varepsilon \\
& =\sum_{k=0}^{d-1} \int_{0}^{\infty} \kappa_{k} \chi_{[0, g / x)}(\varepsilon) x^{-k} \varepsilon^{d-k} \psi(\varepsilon) d \varepsilon-\int_{0}^{\infty} \kappa_{d} \chi_{[g / x, \infty)}(\varepsilon) x^{-d} \psi(\varepsilon) d \varepsilon
\end{aligned}
$$

\footnotetext{
${ }^{7}$ Indeed, $V_{\mathcal{T}}(\varepsilon)$ is clearly continuous and bounded (by the total volume of the spray); hence it defines a locally integrable function on $(0, \infty)$.
} 


$$
\begin{aligned}
& =\sum_{k=0}^{d-1} \kappa_{k} x^{-k} \int_{0}^{g / x} \varepsilon^{d-k} \psi(\varepsilon) d \varepsilon-\kappa_{d} x^{-d} \int_{g / x}^{\infty} \psi(\varepsilon) d \varepsilon \\
& =\sum_{k=0}^{d} \varphi_{k}(x),
\end{aligned}
$$

where, for $x>0$, we have introduced

$$
\varphi_{k}(x):= \begin{cases}\kappa_{k} x^{-k} \int_{0}^{g / x} \varepsilon^{d-k} \psi(\varepsilon) d \varepsilon, & 0 \leq k \leq d-1, \\ \kappa_{k} x^{-k} \int_{\infty}^{g / x} \psi(\varepsilon) d \varepsilon, & k=d,\end{cases}
$$

in the last line. Caution: $\varphi_{k}$ is a function of $x$, whereas $\psi$ is a function of $\varepsilon$.

Putting (7.8) into (7.7), we obtain

$$
\left\langle V_{\mathcal{T}}, \psi\right\rangle=\left\langle\eta_{\mathfrak{s}}, \sum_{k=0}^{d} \varphi_{k}\right\rangle=\sum_{k=0}^{d}\left\langle\eta_{\mathfrak{s}}, \varphi_{k}\right\rangle .
$$

To apply Theorem 6.4, we must first check that the functions $\varphi_{k}$ satisfy the hypotheses (6.9)-(6.10). Recall that $\psi \in C_{c}^{\infty}(0, \infty)$.

For $k<d$, (6.9) is satisfied because for large $x$, the corresponding integral in (7.9) is taken over a set outside the (compact) support of $\psi$. This gives $\varphi_{k}(x)=0$ for sufficiently large $x$, and it is clear that, a fortiori, the $n^{\text {th }}$ derivative of $\varphi_{k}$ satisfies

$$
\varphi_{k}^{(n)}(x)=O\left(x^{-n-D-\delta}\right) \quad \text { for } x \rightarrow \infty .
$$

To see that (6.10) is satisfied, note that $\psi$ vanishes for $x$ sufficiently large and thus

$$
\varphi_{k}(x)=\kappa_{k} x^{-k} \int_{0}^{\infty} \varepsilon^{d-k} \psi(\varepsilon) d \varepsilon \quad \text { for } x \approx 0,
$$

i.e., $\varphi_{k}(x)=a_{k} x^{-k}$ for all small enough $x>0$, where $a_{k}$ is the constant

$$
a_{k}:=\kappa_{k} \int_{0}^{\infty} \varepsilon^{d-k} \psi(\varepsilon) d \varepsilon=\kappa_{k} \tilde{\psi}(d-i+1)=\lim _{x \rightarrow 0^{+}} x^{k} \varphi_{k}(x) .
$$

Here $\tilde{\psi}$ is the Mellin transform of $\psi$, as in (6.14).

Thus, the expansion (6.10) for the test function $\varphi_{k}$ consists of only one term, and for each $n=0,1, \ldots, N,{ }^{8}$

$$
\varphi_{k}^{(n)}(x)=\frac{d^{n}}{d x^{n}}\left[a_{k} x^{-k}\right]=O\left(x^{-n-k}\right) \quad \text { for } x \rightarrow 0^{+} .
$$

A key point is that since $\psi$ is smooth, (7.11) and (7.13) will hold for each $n=0,1, \ldots, N$, as required by Theorem 6.4. Since the expansion of $\varphi_{k}$ has only one term, the only $\alpha$ in the sum is $\alpha=k$. Thus $a_{k}$ is the constant corresponding to $a_{\alpha}$ in (6.10).

\footnotetext{
${ }^{8}$ Recall that $\eta_{\mathfrak{s}}$ is languid of order $\varpi$ and that $N=[\varpi]+2$ in the hypotheses of Theorem 6.4.
} 
Applying Theorem 6.4 in the case when $k<d$, (6.11) becomes

$$
\begin{gathered}
\left\langle\eta_{\mathfrak{s}}, \varphi_{k}\right\rangle=\sum_{\omega \in \mathcal{D}_{\mathfrak{s}}(W)} \operatorname{res}\left(\zeta_{\mathfrak{s}}(s) \tilde{\varphi}_{k}(s) ; \omega\right)+\left\{a_{k} \zeta_{\mathfrak{s}}(k)\right\}_{k \in W \backslash \mathcal{D}_{\mathfrak{s}}} \\
+\frac{1}{2 \pi \AA} \int_{S} \zeta_{\mathfrak{s}}(s) \tilde{\varphi}_{k}(s) d s,
\end{gathered}
$$

where the term in braces is to be included iff $k \in W \backslash \mathcal{D}_{\mathfrak{s}}$. Here and henceforth, $\tilde{\varphi}_{k}$ denotes the Mellin transform of $\varphi_{i}$ given by

$$
\tilde{\varphi}_{k}(s)=\int_{0}^{\infty} x^{s-1} \varphi_{k}(x) d x .
$$

The case when $k=d$ is similar (or antisimilar). The compact support of $\psi$ again gives

$$
\varphi_{d}(x)=\kappa_{d} x^{-d} \int_{\infty}^{0} \psi(\varepsilon) d \varepsilon, \quad \text { for } x \rightarrow \infty,
$$

so that for some positive constant $c$, and for all sufficiently large $x$, we have $\kappa_{d}(x)=c x^{-d}$. Hence

$$
\varphi_{d}^{(n)}(x)=O\left(x^{-n-d}\right) \text { for } x \rightarrow \infty, \forall n \geq 0,
$$

and (6.9) is satisfied. For very small $x$, the integral in the definition of $\kappa_{d}(x)$ is taken over an interval outside the support of $\psi$, and hence $\kappa_{d}(x)=0$ for $x \approx 0$. Then clearly (6.10) is satisfied:

$$
\varphi_{d}^{(n)}(x)=0 \quad \text { for } x \rightarrow 0^{+}, \forall n \geq 0 .
$$

An immediate consequence of (7.18) is that for $k=d$ in (7.12), the constant term is

$$
a_{d}=\lim _{x \rightarrow 0} x^{d} \varphi_{d}(x)=0
$$

and compared with (7.14) we have one term less in

$$
\left\langle\eta_{\mathfrak{s}}, \varphi_{d}\right\rangle=\sum_{\omega \in \mathcal{D}_{\mathfrak{s}}(W)} \operatorname{res}\left(\zeta_{\mathfrak{s}}(s) \tilde{\varphi}_{d}(s) ; \omega\right)+\frac{1}{2 \pi \AA} \int_{S} \zeta_{\mathfrak{s}}(s) \tilde{\varphi}_{d}(s) d s .
$$

As in (7.15), denote the Mellin transform of the function $\psi$ by $\tilde{\psi}$ and compute

$$
\begin{aligned}
\tilde{\varphi}_{k}(s)=\int_{0}^{\infty} x^{s-1} \varphi_{k}(x) d x & =\kappa_{k} \int_{0}^{\infty} x^{s-k-1} \int_{0}^{g / x} \varepsilon^{d-k} \psi(\varepsilon) d \varepsilon d x \\
& =\kappa_{k} \int_{0}^{\infty}\left(\int_{0}^{g / \varepsilon} x^{s-k-1} d x\right) \varepsilon^{d-k} \psi(\varepsilon) d \varepsilon \\
& =\frac{\kappa_{k}}{s-k} \int_{0}^{\infty} g^{s-k} \varepsilon^{k-s} \varepsilon^{d-k} \psi(\varepsilon) d \varepsilon \\
& =g^{s-k} \frac{\kappa_{k}}{s-k} \tilde{\psi}(d-s+1) .
\end{aligned}
$$

By a similar calculation,

$$
\tilde{\varphi}_{d}(s)=g^{s-d} \frac{\kappa_{d}}{s-d} \tilde{\psi}(d-s+1)
$$


Note that for $0 \leq k<d-1$, (7.21) is valid for $\operatorname{Re} s>k$, and for $k=d$, (7.22) is valid for $\operatorname{Re} s<k$. Thus both are valid in the strip $d-1<\operatorname{Re} s<d$, and hence by analytic (meromorphic) continuation, they are valid everywhere in $\mathbb{C}$. Indeed, by Corollary A.4, $\tilde{\psi}$ is entire.

We return to the evaluation of (7.10), applying Theorem 6.4 to find the action of $\eta_{\mathfrak{s}}$ on the test function $\varphi_{k}$, for $k=0, \ldots, d$. Substituting (7.21) and (7.22) into (7.14) gives

$$
\begin{aligned}
\left\langle\eta_{\mathfrak{s}}, \varphi_{k}\right\rangle= & \sum_{\omega \in \mathcal{D}_{\mathfrak{s}}(W)} \operatorname{res}\left(\zeta_{\mathfrak{s}}(s) \frac{g^{s-k} \kappa_{k}}{s-k} \tilde{\psi}(d-s+1) ; \omega\right) \\
& +\left\{a_{k} \zeta_{\mathfrak{s}}(k)\right\}_{k \in W \backslash \mathcal{D}_{\mathfrak{s}}}+\left\langle\mathcal{R}_{k}, \psi\right\rangle,
\end{aligned}
$$

where $\mathcal{R}_{k}$ is defined by

$$
\left\langle\mathcal{R}_{k}, \psi\right\rangle:=\frac{1}{2 \pi \AA} \int_{S} \zeta_{\mathfrak{s}}(s) \tilde{\varphi}_{k}(s) d s .
$$

Substituting (7.23) into (7.10), we obtain

$$
\begin{aligned}
\langle V(\varepsilon), \psi\rangle= & \sum_{k=0}^{d} \sum_{\omega \in \mathcal{D}_{\mathfrak{s}}(W)} \operatorname{res}\left(\zeta_{\mathfrak{s}}(s) \frac{g^{s-k} \kappa_{k}}{s-k} \tilde{\psi}(d-s+1) ; \omega\right) \\
& +\sum_{k=0}^{d}\left\{a_{k} \zeta_{\mathfrak{s}}(k)\right\}_{k \in W \backslash \mathcal{D}_{\mathfrak{s}}}+\sum_{k=0}^{d}\left\langle\mathcal{R}_{k}(\varepsilon), \psi(\varepsilon)\right\rangle .
\end{aligned}
$$

Recall from (7.19) that the $d^{\text {th }}$ term is $a_{d}=0$, so the top term of the second sum vanishes. Note that at each such $k$ we have a residue

$$
\begin{aligned}
\operatorname{res}\left(\zeta_{\mathfrak{s}}(s) \frac{g^{s-k} \kappa_{k}}{s-k} \tilde{\psi}(d-s+1) ; k\right) & =\kappa_{k} \lim _{s \rightarrow k} \zeta_{\mathfrak{s}}(s) g^{s-k} \tilde{\psi}(d-s+1) \\
& =\kappa_{k} \zeta_{\mathfrak{s}}(k) \tilde{\psi}(d-i+1) \\
& =a_{k} \zeta_{\mathfrak{s}}(k) .
\end{aligned}
$$

Since the terms of the second sum of (7.25) are only included for $k \in W \backslash \mathcal{D}_{\mathfrak{s}}(W)$, we can use (7.3) and (7.26) to combine the last two sums of (7.25) without losing or duplicating terms:

$$
\langle V(\varepsilon), \psi\rangle=\sum_{\omega \in \mathcal{D}_{\mathcal{T}}(W)} \operatorname{res}\left(\tilde{\psi}(d-s+1) \zeta_{\mathfrak{s}}(s) \sum_{k=0}^{d} \frac{g^{s-k} \kappa_{k}}{s-k} ; \omega\right)+\langle\mathcal{R}(\varepsilon), \psi(\varepsilon)\rangle,
$$

where $\mathcal{R}(\varepsilon):=\sum_{k=0}^{d} \mathcal{R}_{k}(\varepsilon)$. This may also be written as the distribution

$$
V(\varepsilon)=\sum_{\omega \in \mathcal{D}_{\mathfrak{s}}(W)} \operatorname{res}\left(\varepsilon^{d-s} \zeta_{\mathfrak{s}}(s) \sum_{k=0}^{d} \frac{g^{s-k} \kappa_{k}}{s-k} ; \omega\right)+\mathcal{R}(\varepsilon) .
$$

This completes the proof of (7.4). All that remains is the verification of the expression (7.5) for the error term, and error estimate (7.6). Due to their technical and specialized nature, we leave the proofs of (7.5) and (7.6) to Appendix B. 


\section{Extensions and Consequences: the Tube Formula for Self-Similar Tilings}

Recall from Sect. 5.4 that the results of Sect. 8 may easily be extended to multiple generators simply by taking the corresponding finite sum. The next corollary indicates that when $\zeta_{\mathfrak{s}}$ is strongly languid, one may take $W=\mathbb{C}$ in the previous theorem and the error term will vanish identically.

Corollary 8.1 (Tube Formula for Strongly Languid Fractal Sprays) Let $\mathcal{T}$ be a fractal spray on the monophase generator $G$, and additionally assume that $\zeta_{\mathfrak{s}}$ is strongly languid, and hence that $W=\mathbb{C}$. Then

$$
V_{\mathcal{T}}(\varepsilon)=\sum_{\omega \in \mathcal{D}_{\mathcal{T}}} \operatorname{res}\left(\zeta_{\mathcal{T}}(\varepsilon, s) ; \omega\right)
$$

where $\mathcal{D}_{\mathcal{T}}=\mathcal{D}_{\mathcal{T}}(\mathbb{C})$ is the set of complex dimensions of $\mathcal{T}$, as in (7.3).

Proof This is immediate upon combining Theorem 6.5 (the extended distributional formula without error term) with the proof of Theorem 7.4. One finds that $\mathcal{R}_{k} \equiv 0$ for each $k=$ $0,1, \ldots, d$ in (7.24) and thus $\mathcal{R} \equiv 0$ in (7.27).

Remark 8.2 (Reality Principle) The nonreal complex dimensions appear in complex conjugate pairs and produce terms with coefficients which are also complex conjugates, in the general tube formula for fractal sprays. This ensures that formulas (7.4) and (8.1)-(8.2) are real-valued.

\subsection{The Self-Similar Case}

Self-similar strings automatically satisfy the more stringent hypothesis of being strongly languid, as in Definition 6.3. This automatically entails that Corollary 8.1 holds, ${ }^{9}$ so the window may be taken to be all of $\mathbb{C}$ and the error term vanishes identically, i.e., $\mathcal{R}(\varepsilon) \equiv 0$. Hence Theorem 7.4 may be strengthened for self-similar tilings as in Theorem 8.3.

Theorem 8.3 (Tube Formula for Self-Similar Tilings) Let $\mathcal{T}=\left\{\Phi_{w} G\right\}$, be a self-similar tiling with pluriphase generator $G$ and geometric zeta function $\zeta_{\mathcal{T}}$. Then the d-dimensional volume of the inner tubular neighbourhood of $\mathcal{T}$ is given by the following distributional explicit formula:

$$
V_{\mathcal{T}}(\varepsilon)=\sum_{\omega \in \mathcal{D}_{\mathcal{T}}} \operatorname{res}\left(\zeta_{\mathcal{T}}(\varepsilon, s) ; \omega\right)
$$

where $\mathcal{D}_{\mathcal{T}}=\mathcal{D}_{\mathcal{T}}(\mathbb{C})=\mathcal{D}_{\mathfrak{s}}(\mathbb{C}) \cup\{0,1, \ldots, d-1\}$ is the set of complex dimensions of $\mathcal{T}$.

Proof The proof follows [30, Sect. 6.4]. According to Theorem 4.7, the scaling zeta function of a self-similar tiling has the form

$$
\zeta_{\mathfrak{s}}(s)=\frac{1}{1-\sum_{j=1}^{J} r_{j}^{s}} .
$$

\footnotetext{
${ }^{9}$ This is essentially because Theorem 6.4 and Theorem 6.6 hold without error term. This is discussed further in [30, Theorem 5.27], and the end of [30, Theorem 8.1]. A general discussion of the strongly languid case may be found in [30, Definition 5.3], and an argument showing that all self-similar strings are strongly languid is given in [30, Sect. 6.4].
} 
Let $r_{J}$ be the smallest scaling ratio. Then from

$$
\left|\zeta_{\mathfrak{s}}(s)\right| \ll\left(\frac{1}{r_{J}}\right)^{-|\sigma|} \quad \text { as } \sigma=\operatorname{Re}(s) \rightarrow-\infty,
$$

we deduce that $\zeta_{\mathcal{T}}$ is strongly languid and therefore apply Corollary 8.1. This argument follows from the analogous ideas regarding self-similar strings, which may be found in [30, Sect. 8.4].

Remark 8.4 Theorem 8.3 provides a higher-dimensional counterpart of the tube formula obtained for self-similar strings in [30, Sect. 8.4]. It should be noted that Theorem 8.3 applies to a slightly smaller class of test functions than Theorem 7.4. Indeed, the support of the test functions must be bounded away from 0 by $\mu_{d}(C) g / r_{J}$, where $C=[F]$ is the hull of the attractor (as in Sect. 2), $g$ is the smallest generating inradius (as in (3.6)), and $r_{J}$ is the smallest scaling ratio of $\Phi$ (as in (2.1)). This technicality is discussed further in [30], Definition 5.3 and Theorem 5.27, Sect. 6.4, and Theorem 8.1.

Corollary 8.5 (Measurability and the Lattice/Nonlattice Dichotomy) Under mild conditions on the residues of $\zeta_{\tau}$, a self-similar tiling is Minkowski measurable if and only if it is nonlattice.

Sketch of Proof We define a self-similar tiling $\mathcal{T}$ to be Minkowski measurable iff

$$
0<\lim _{\varepsilon \rightarrow 0^{+}} V_{\mathcal{T}}(\varepsilon) \varepsilon^{-(d-D)}<\infty,
$$

i.e., if the limit in (8.3) exists and takes a value in $(0, \infty)$. A tiling has infinitely many complex dimensions with real part $D$ iff it is lattice type, as mentioned in Sect. 4.3. Furthermore, all the poles with real part $D$ are simple in that case. A glance at (8.5) then shows that $V_{\mathcal{T}}(\varepsilon) \varepsilon^{-(d-D)}$ is a sum containing infinitely many purely oscillatory terms $c_{\omega} \varepsilon^{8 n \mathbf{p}}, n \in \mathbb{Z}$, where $\mathbf{p}$ is some fixed period. Thus, the limit (8.3) cannot exist; see also [30, Sect. 8.4.2]. ${ }^{10}$ Conversely, the tiling is nonlattice iff $D$ is the only complex dimension with real part $D$. In this case, $D$ is simple and no term in the sum $V_{\mathcal{T}}(\varepsilon) \varepsilon^{-(d-D)}$ is purely oscillatory; thus the tiling $\mathcal{T}$ is measurable. See also [30, Sect. 8.4.4].

Note added in proof Please see Remark 10.6 for the "mild conditions" mentioned in the statement of Corollary 8.5, and the scope of Remark 8.6 and Remark 8.12.

Remark 8.6 In [30, Sects. 8.3-8.4], it is shown that a self-similar fractal string (i.e., a 1-dimensional self-similar tiling) is Minkowski measurable if and only if it is nonlattice. Gatzouras showed in [8] that nonlattice self-similar subsets of $\mathbb{R}^{d}$ are Minkowski measurable, thereby extending to higher dimensions a result in $[3,19]$ and partially proving the geometric part of [19, Conjecture 3]. The previous result gives a complete characterization of self-similar tilings in $\mathbb{R}^{d}$ as nonlattice if and only if they are Minkowski measurable. With the exception of Remark 9.2, each of the examples discussed in Sect. 9 is lattice and hence not Minkowski measurable. Our results, however, apply to nonlattice tilings as well. A more detailed proof of Corollary 8.5 is possible via truncation, by using the screen and window technique of [30, Theorem 5.31 and Theorem 8.36].

\footnotetext{
${ }^{10}$ It is shown in [30] that infinitely many coefficients $c_{\omega}$ are nonzero for $\operatorname{Re} \omega=D$.
} 
The following corollary of Theorem 8.3 will be used in Sect. 9 .

Corollary 8.7 If, in addition to the hypotheses of Theorem $8.3, G$ is monophase and $\zeta_{\mathcal{T}}(s)$ has only simple poles, then

$$
V_{\mathcal{T}}(\varepsilon)=\sum_{\omega \in \mathcal{D}_{\mathfrak{s}}} \sum_{k=0}^{d} \operatorname{res}\left(\zeta_{\mathfrak{s}}(s) ; \omega\right) \varepsilon^{d-\omega} \frac{g^{\omega-k}}{\omega-k} \kappa_{k}+\sum_{k=0}^{d-1} \kappa_{k} \zeta_{\mathfrak{s}}(k) \varepsilon^{d-k} .
$$

It is not an error that the first sum extends to $d$ in (8.4), while the second stops at $d-1$; see (7.19). Note that in Corollary 8.7, $\mathcal{D}_{\mathfrak{s}}$ does not contain any integer $k=0,1, \ldots, d-1$, because this would imply that $\zeta_{\mathcal{T}}$ has a pole of multiplicity at least 2 at such an integer. In general, at most one integer can possibly be a pole of $\zeta_{\mathfrak{s}}$; see Sect. 4.3.

Remark 8.8 For self-similar tilings satisfying the hypotheses of Corollary 8.7, it is clear that the general form of the tube formula is

$$
V_{\mathcal{T}}(\varepsilon)=\sum_{\omega \in \mathcal{D}_{\mathcal{T}}} c_{\omega} \varepsilon^{d-\omega}
$$

where for each fixed $\omega \in \mathcal{D}_{\mathfrak{s}}$,

$$
c_{\omega}:=\operatorname{res}\left(\zeta_{\mathfrak{s}}(s) ; \omega\right) \sum_{k=0}^{d} \frac{g^{\omega-k}}{\omega-k} \kappa_{k} .
$$

Note that when $\omega=k \in\{0,1, \ldots, d-1\}$, one has $c_{\omega}=c_{k}=\zeta_{\mathfrak{s}}(k) \kappa_{k}$.

Remark 8.9 The oscillatory nature of the geometry of $\mathcal{T}$ is apparent in (8.5). In particular, the existence of the limit in (8.3) can be determined in the nonlattice case by examining (8.5) and $\mathcal{D}_{\mathcal{T}}$.

Remark 8.10 In the literature regarding the 1-dimensional case [5, 28, 30], the terms "gaps" and "multiple gaps" have been used where we have used "generators".

Remark 8.11 (Comparison of $V_{\mathcal{T}}$ with the Steiner Formula) In the trivial situation when the spray consists only of finitely many scaled copies of a monophase generator (so the scaling measure $\eta_{\mathfrak{s}}$ is supported on a finite set), the zeta function $\zeta_{\mathfrak{s}}$ will have no poles in $\mathbb{C}$. Therefore, the tube formula becomes a sum over only the numbers $0,1, \ldots, d-1$ (recall from (7.19) that $a_{d}=0$, so the $d^{\text {th }}$ summand vanishes), for which the residues simplify greatly as in (7.26). In this case, $\zeta_{\mathfrak{s}}(k)=\rho_{1}^{k}+\cdots+\rho_{J}^{k}$, so each residue from (7.26) becomes a finite sum

$$
\begin{aligned}
\zeta_{\mathfrak{s}}(k) \kappa_{k}(\varepsilon) & =\rho_{1}^{k} \kappa_{k} \varepsilon^{d-k}+\cdots+\rho_{J}^{k} \kappa_{k} \varepsilon^{d-k} \\
& =\kappa_{k}\left(r_{w_{1}} G\right) \varepsilon^{d-k}+\cdots+\kappa_{k}\left(r_{w_{J}} G\right) \varepsilon^{d-k}
\end{aligned}
$$

where $J$ is the number of scaled copies of the generator $G$, and $r_{w_{j}}$ is the corresponding scaling factor. Thus, for each $j=1, \ldots, J$, we obtain a monophase formula for the scaled basic shape $r_{w_{j}} G$. 
Remark 8.12 [Combining the results of this paper with [30].] Since, as was noted in Sect. 4.3 , the structure of the scaling complex dimensions of a self-similar tiling in $\mathbb{R}^{d}$ is the same as in the 1-dimensional case, we could state an analogue of each of the theorems given in [30, Sect. 8.4], whether in the lattice case ([30, Sect. 8.4.2]) or in the nonlattice case ([30, Sect. 8.4.4]). In particular, we can apply Theorem 7.4 with a suitable window $W$ (and use the Diophantine approximation techniques of [30, Chap. 3]) in order to obtain the exact higher-dimensional analogues of the tube formulas with error term stated in [30, Corollary 8.27] and [30, Theorem 8.37 and Eqn. (8.71)], in the lattice and nonlattice case, respectively. Finally, in the lattice case, following [30, Sect. 8.4.3], even though the self-similar tiling is not Minkowski measurable, we could calculate its 'average Minkowski content' (cf. [30, Definition 8.29] with 1 replaced by $d$ ) and obtain the $d$-dimensional analogue of [30, Theorem 8.30]. In order to avoid redundancies, we will avoid formulating explicitly any of these consequences of our results in this paper. An attentive reader of [30] should easily be able to combine that material with the present results and obtain such useful corollaries.

\subsection{Recovering the Tube Formula for Fractal Strings}

In this section, we discuss the 1-dimensional tube formula of Theorem 6.6 which is true for general (i.e., not necessarily self-similar) fractal strings and which can be recovered from Theorem 7.4. Suppose $\mathcal{L}=\left\{\ell_{n}\right\}_{n=1}^{\infty}$ is a languid fractal string with associated measure $\eta_{\mathcal{L}}=$ $\sum_{n=1}^{\infty} \delta_{1 / \ell_{n}}$, as in (4.2), and associated zeta function $\zeta_{\mathcal{L}}=\sum_{n=1}^{\infty} \ell_{n}^{s}$, as in (4.1). Considering the string now as a tiling, write $\mathcal{L}$ as $L=\left\{L_{n}\right\}_{n=1}^{\infty}$ to emphasize the fact that we are thinking of it as a spray instead of as a string. Take the spray $L$ to have as its single generator the interval $G=(0,2)$, so that $L$ has inradii $\rho_{n}=\frac{1}{2} \ell_{n}$ and that the length of $\rho_{n} G$ is $\ell_{n}$. Now the scaling measure is $\eta_{\mathfrak{s}}=\sum_{n=1}^{\infty} \delta_{2 / \ell_{n}}$ and the scaling zeta function is

$$
\zeta_{\mathfrak{s}}(s)=\sum_{n=1}^{\infty}\left(\frac{\ell_{n}}{2}\right)^{s}=2^{-s} \zeta_{\mathcal{L}}(s)
$$

The generator is clearly monophase with $\kappa_{0}=2$ and $\kappa_{1}=-2 g$ :

$$
\gamma_{G}(x, \varepsilon)= \begin{cases}2 \varepsilon, & \varepsilon \leq g / x, \\ 2 g / x, & \varepsilon \geq g / x .\end{cases}
$$

One obtains the tubular zeta function of the (1-dimensional) tiling $L$ as

$$
\zeta_{L}(\varepsilon, s)=\varepsilon^{1-s} \zeta_{\mathfrak{s}}(s) \sum_{k=0}^{1} \frac{\kappa_{k}}{s-k}=\varepsilon^{1-s} 2^{-s} \zeta_{\mathfrak{s}}(s)\left(\frac{2}{s}-\frac{2}{s-1}\right)=\frac{\zeta_{\mathcal{L}}(s)(2 \varepsilon)^{1-s}}{s(1-s)}
$$

by substituting in (8.7) in the last step. Then from Theorem 7.4 we exactly recover the tube formula $V_{L}(\varepsilon)=V_{\mathcal{L}}(\varepsilon)$ (and its error term) as given by Theorem 6.6. Note that $\mathcal{D}_{\mathfrak{s}}=\mathcal{D}_{\mathcal{L}}$ by (8.7).

Remark 8.13 In addition to recovering a previously known formula, we also gain a geometric interpretation of the terms appearing in the 1-dimensional tube formula (6.16), in view of the previous computation. In particular, one sees that the linear term $\left\{2 \varepsilon \zeta_{\mathfrak{s}}(0)\right\}$ has 
a geometric interpretation in terms of the inner Steiner formula for an interval, and can be dissected as

$$
2 \varepsilon \zeta_{\mathfrak{s}}(0)=\kappa_{0}(G) \varepsilon^{1-0} \zeta_{\mathfrak{s}}(0)=(-2) \mu_{k}(G) \varepsilon^{d-k} \zeta_{\mathfrak{s}}(k),
$$

where $k=0$ and $d=1$. Note that $\mu_{0}(G)=-1$ is the Euler characteristic of an open interval. This should be discussed further in [23].

\section{Tube Formula Examples}

Although Remark 9.2 discusses how one may construct nonlattice examples, the other examples chosen in this section are lattice self-similar tilings, in the sense of Sect. 4.3. Also, all examples in this section have monophase generators in the sense of Definition 5.2, as is verified in each case. The pentagasket of Example 9.4 is the only example given here of a self-similar tiling with multiple generators.

Moreover, the scaling zeta function $\zeta_{\mathfrak{s}}$ of each example has only simple poles, with a single line of complex dimensions distributed periodically on the line $\operatorname{Re} s=D$. Thus, the tube formula may be substantially simplified via Corollary 8.7.

\subsection{The Cantor Tiling}

The Cantor tiling $\mathcal{C}$ (called the Cantor string in [30], Sect. 1.1.2 and Sect. 2.3.1) is constructed via the self-similar system

$$
\Phi_{1}(x)=\frac{x}{3}, \quad \Phi_{2}(x)=\frac{x+2}{3} .
$$

The associated self-similar set $F$ is the classical ternary Cantor set, so $d=1$ and we have one scaling ratio $r=\frac{1}{3}$, and one generator $G=\left(\frac{1}{3}, \frac{2}{3}\right)$ with generating inradius $g=\frac{1}{6}$. The corresponding self-similar string has inradii $\rho_{m}=g r^{m}$ with multiplicity $2^{m}, m=0,1,2, \ldots$, so the scaling zeta function is

$$
\zeta_{\mathfrak{s}}(s)=\frac{1}{1-2 \cdot 3^{-s}},
$$

and the scaling complex dimensions are

$$
\mathcal{D}_{\mathfrak{s}}=\{D+\stackrel{\circ}{ } n \mathbf{p}: n \in \mathbb{Z}\} \quad \text { for } D=\log _{3} 2, \mathbf{p}=\frac{2 \pi}{\log 3} .
$$

We note that $\zeta_{\mathfrak{s}}(0)=-1$ and apply (8.9) from the previous section to recover the following tube formula for $\mathcal{C}$ (as obtained in [30], Sect. 1.1.2):

$$
V_{\mathcal{C}}(\varepsilon)=\frac{1}{2 \log 3} \sum_{n \in \mathbb{Z}} \frac{(2 \varepsilon)^{1-D-i n \mathbf{p}}}{(D+i n \mathbf{p})(1-D-i n \mathbf{p})}-2 \varepsilon .
$$

Alternatively, this may be written as a series in $\left(\frac{\varepsilon}{g}\right)$ as

$$
V_{\mathcal{C}}(\varepsilon)=\frac{1}{3 \log 3} \sum_{n \in \mathbb{Z}}\left(\frac{1}{D+i n \mathbf{p}}-\frac{1}{D-1+\AA n \mathbf{p}}\right)\left(\frac{\varepsilon}{g}\right)^{1-D-8 n \mathbf{p}}-2 \varepsilon,
$$

with $g=\frac{1}{6}, D=\log _{3} 2$, and $\mathbf{p}=2 \pi / \log 3$. It is this form of the tube formula which is closer in appearance to the following examples. 


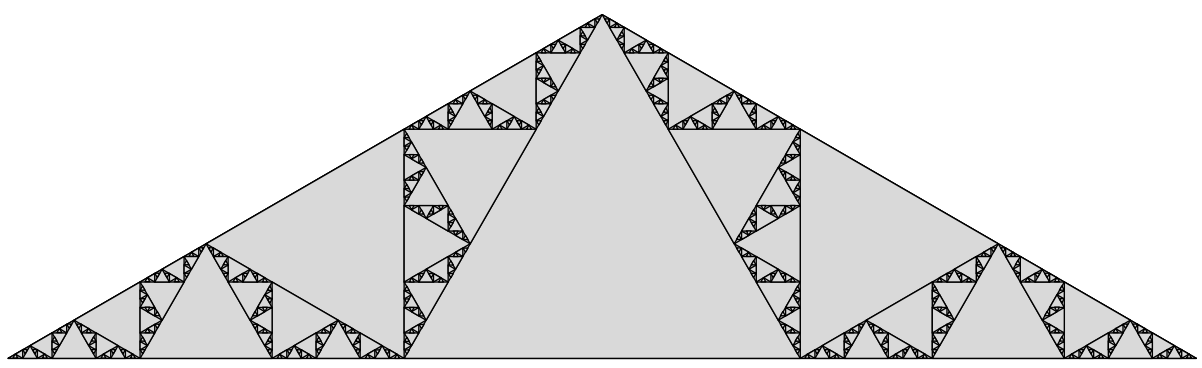

Fig. 3 The Koch tiling $\mathcal{K}$

\subsection{The Koch Tiling}

The standard Koch tiling $\mathcal{K}$ (see Fig. 3, along with Fig. 1 of Sect. 2) is constructed via the self-similar system

$$
\Phi_{1}(z):=\xi \bar{z} \quad \text { and } \quad \Phi_{2}(z):=(1-\xi)(\bar{z}-1)+1,
$$

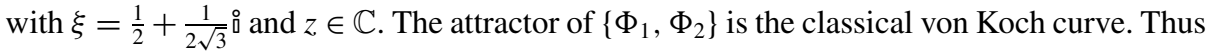
$\mathcal{K}$ has one scaling ratio $r=|\xi|=1 / \sqrt{3}$, and one generator $G$ : an equilateral triangle of side length $\frac{1}{3}$ and generating inradius $g=\frac{\sqrt{3}}{18}$. This tiling has inradii $\rho_{m}=g r^{m}$ with multiplicity $2^{m}$, where $m=0,1,2, \ldots$, so the scaling zeta function is

$$
\zeta_{\mathfrak{s}}(s)=\frac{1}{1-2 \cdot 3^{-s / 2}}
$$

and the scaling complex dimensions are

$$
\mathcal{D}_{\mathfrak{s}}=\left\{D+\AA^{n} n \mathbf{p}: n \in \mathbb{Z}\right\} \quad \text { for } D=\log _{3} 4, \mathbf{p}=\frac{4 \pi}{\log 3} .
$$

By inspection, a tile with inradius $1 / x$ will have tube formula

$$
\gamma_{G}(x, \varepsilon)= \begin{cases}3^{3 / 2}\left(-\varepsilon^{2}+2 \varepsilon x\right), & \varepsilon \leq 1 / x, \\ 3^{3 / 2} x^{2}, & \varepsilon \geq 1 / x .\end{cases}
$$

For fixed $x,(9.8)$ is clearly continuous at $\varepsilon=0^{+}$. Thus we have

$$
\begin{aligned}
\zeta_{\mathfrak{s}}(s) & =\frac{1}{1-2 \cdot 3^{-s / 2}} \quad \text { and } \\
\kappa_{0} & =-3^{3 / 2}, \quad \kappa_{1}=2 \cdot 3^{3 / 2}, \quad \kappa_{2}=-3^{3 / 2} .
\end{aligned}
$$

Now applying (8.4), the tube formula for the Koch tiling $\mathcal{K}$ is

$$
\begin{aligned}
V_{\mathcal{K}}(\varepsilon)= & 3^{3 / 2} g^{2} \sum_{\omega \in \mathcal{D}_{\mathfrak{s}}} \operatorname{res}\left(\frac{1}{1-2 \cdot 3^{-s / 2}} ; \omega\right)\left(-\frac{1}{\omega}+\frac{2}{\omega-1}-\frac{1}{\omega-2}\right)\left(\frac{\varepsilon}{g}\right)^{2-\omega} \\
& +\frac{g}{2} \zeta_{\mathfrak{s}}(0) \operatorname{res}\left(-\frac{1}{s} ; 0\right)\left(\frac{\varepsilon}{g}\right)^{2-0}+\frac{g}{2} \zeta_{\mathfrak{s}}(1) \operatorname{res}\left(\frac{2}{s-1} ; 1\right)\left(\frac{\varepsilon}{g}\right)^{2-1}
\end{aligned}
$$




$$
\begin{aligned}
= & \frac{g}{\log 3} \sum_{n \in \mathbb{Z}}\left(-\frac{1}{D+i n \mathbf{p}}+\frac{2}{D-1+i n \mathbf{p}}-\frac{1}{D-2+i n \mathbf{p}}\right)\left(\frac{\varepsilon}{g}\right)^{2-D-i n \mathbf{p}} \\
& +3^{3 / 2} \varepsilon^{2}+\frac{1}{1-2 \cdot 3^{-1 / 2}} \varepsilon,
\end{aligned}
$$

where $D=\log _{3} 4, g=\frac{\sqrt{3}}{18}$ and $\mathbf{p}=\frac{4 \pi}{\log 3}$ as before.

Remark 9.1 In [21], a tube formula was obtained for the Koch curve itself (rather than for the tiling associated with it) and the possible complex dimensions of this curve were inferred to be

$$
\mathcal{D}_{\mathcal{K}_{\star}}=\{D+\stackrel{\circ}{ } \mathbf{p}: n \in \mathbb{Z}\} \cup\{0+\stackrel{\circ}{ } n \mathbf{p}: n \in \mathbb{Z}\},
$$

where $D=\log _{3} 4$ and $\mathbf{p}=\frac{2 \pi}{\log 3}$. The line of poles above $D$ was expected, ${ }^{11}$ and agrees precisely with the results of this paper. The meaning of the line of poles above 0 is still unclear. A zeta function for the Koch curve was not defined prior to the present paper; all previous reasoning was by analogy with (6.16).

Remark 9.2 (Nonlattice Koch Tilings) By replacing $\xi=\frac{1}{2}+\frac{1}{2 \sqrt{3}}^{\circ}$ in (9.5) with any other complex number satisfying $|\xi|^{2}+|1-\xi|^{2}<1$, one obtains a family of examples of nonlattice self-similar tilings. The tube formula computations parallel the lattice case almost identically. The lattice Koch tilings correspond exactly to those $\xi \in B\left(\frac{1}{2}, \frac{1}{2}\right)$ (the ball of radius $\frac{1}{2}$ centered at $\frac{1}{2} \in \mathbb{C}$ ) for which $\log _{r}|\xi|$ and $\log _{r}|1-\xi|$ are both positive integers, for some fixed $0<r<1$. Further discussion (and illustrations) of nonlattice Koch tilings may be found in [32].

\subsection{The Sierpinski Gasket Tiling}

The Sierpinski gasket tiling $\mathcal{S G}$ (see Fig. 4) is constructed via the system

$$
\Phi_{1}(z):=\frac{1}{2} z, \quad \Phi_{2}(z):=\frac{1}{2} z+\frac{1}{2}, \quad \Phi_{3}(z):=\frac{1}{2} z+\frac{1+i \sqrt{3}}{4},
$$
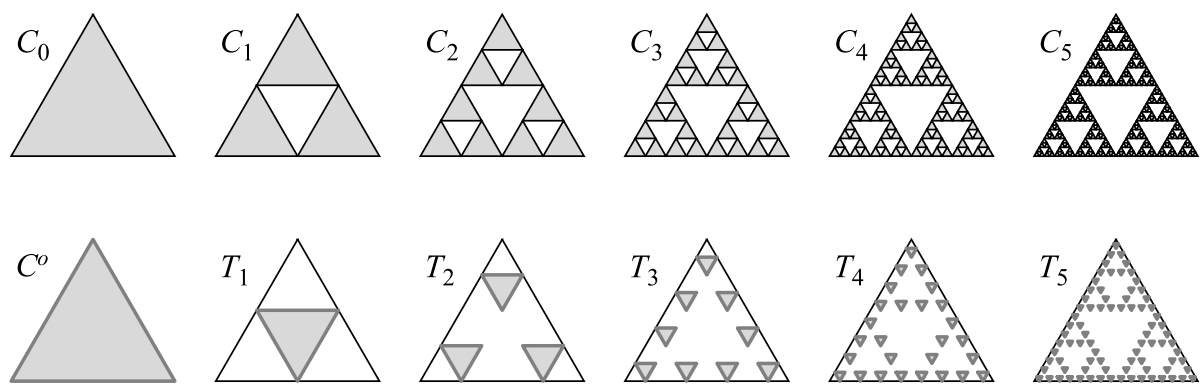

Fig. 4 The Sierpinski gasket tiling

\footnotetext{
${ }^{11}$ This set of complex dimensions was predicted in [27], Sect. 10.3.
} 
which has one common scaling ratio $r=1 / 2$, and one generator $G$ : an equilateral triangle of side length $\frac{1}{2}$ and inradius $g=\frac{1}{4 \sqrt{3}}$. Thus $\mathcal{S G}$ has inradii $\rho_{m}=g r^{m}$ with multiplicity $3^{m}$, $m=0,1,2, \ldots$, so the scaling zeta function is

$$
\zeta_{\mathfrak{s}}(s)=\frac{1}{1-3 \cdot 2^{-s}}
$$

and the scaling complex dimensions are

$$
\mathcal{D}_{\mathfrak{s}}=\{D+\stackrel{i}{ } \mathbf{p}: n \in \mathbb{Z}\} \quad \text { for } D=\log _{2} 3, \mathbf{p}=\frac{2 \pi}{\log 2} .
$$

Aside from $\zeta_{\mathfrak{s}}(s)$, the tube formula calculation for $\mathcal{S G}$ is identical to that for the previous example $\mathcal{K}$ :

$$
\begin{aligned}
& V_{\mathcal{S G}}(\varepsilon)=\frac{\sqrt{3}}{16 \log 2} \sum_{n \in \mathbb{Z}}\left(-\frac{1}{D+i n \mathbf{p}}+\frac{2}{D-1+i n \mathbf{p}}-\frac{1}{D-2+i n \mathbf{p}}\right)\left(\frac{\varepsilon}{g}\right)^{2-D-i n \mathbf{p}} \\
& +\frac{3^{3 / 2}}{2} \varepsilon^{2}-3 \varepsilon \text {. }
\end{aligned}
$$

Remark 9.3 Suppose that for a tiling $\mathcal{T}$, the boundary of the hull intersects the boundary of a generator in at most a finite set: $\left|\partial C \cap \partial G_{q}\right|<\infty$. In this case, the tube formula for the tiling is almost the (exterior) tube formula for the attractor. This is the case for the Sierpinski gasket, and also for the Sierpinksi carpet (in which case the intersection is empty). In fact, the exterior $\varepsilon$-neighbourhood of the Sierpinski gasket curve is obtained by adding the Steiner's formula for $\bar{C}$ :

$$
\operatorname{vol}_{2}\left((\mathcal{S G})_{\varepsilon}\right)=V_{\mathcal{S G}}(\varepsilon)+3 \varepsilon+\pi \varepsilon^{2} .
$$

\subsection{The Pentagasket Tiling}

The pentagasket tiling $\mathcal{P}$ (see Fig. 5) is constructed via the self-similar system defined by the five maps

$$
\Phi_{j}(x)=\frac{3-\sqrt{5}}{2} x+p_{j}, \quad j=1, \ldots, 5,
$$

with common scaling ratio $r=\phi^{-2}$, where $\phi=(1+\sqrt{5}) / 2$ is the golden ratio, and the points $\frac{p_{j}}{1-r}=c_{j}$ form the vertices of a regular pentagon of side length 1 .

The pentagasket $\mathcal{P}$ is an example of multiple generators $G_{q}: G_{1}$ is a regular pentagon and $G_{2}, \ldots, G_{6}$ are congruent isosceles triangles, as seen in $T_{1}$ of Fig. 5. To make the notation more meaningful, we use the subscripts $p, t$ to indicate a pentagon or triangle, respectively. The generating inradius for the pentagon is $g_{p}=\frac{\phi^{2}}{2} \tan \frac{3}{10} \pi$ and the generating inradius for the triangles is $g_{t}=\frac{\phi^{3}}{2} \tan \frac{\pi}{5}$. Thus, $\mathcal{P}$ has inradii $\rho_{m}=g_{q} r^{m}$, for $q=p, t$ and $m=$ $0,1,2, \ldots$, with multiplicity $5^{m}$, so the scaling zeta function is

$$
\zeta_{\mathfrak{s}}(s)=\frac{1}{1-5 \cdot r^{-s}},
$$



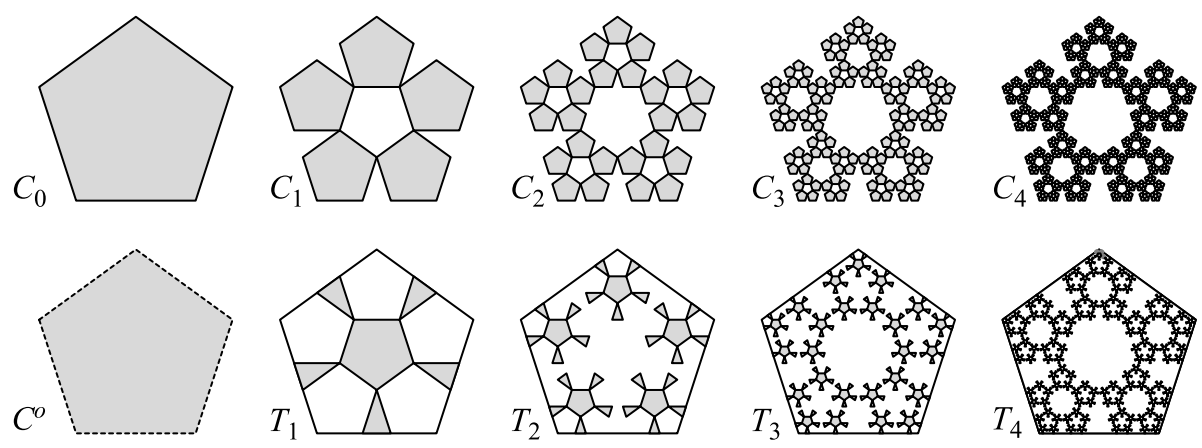

Fig. 5 The pentagasket tiling

Fig. 6 The pentagasket and the golden ratio $\phi$

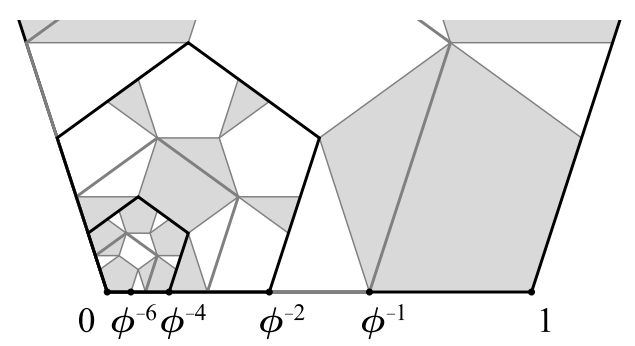

and the scaling complex dimensions are

$$
\mathcal{D}_{\mathfrak{s}}=\left\{D+{ }_{i} n \mathbf{p}: n \in \mathbb{Z}\right\} \quad \text { for } D=\log _{1 / r} 5, \mathbf{p}=\frac{2 \pi}{\log r^{-1}} .
$$

We omit the exercise of finding volumes for the pentagonal and triangular generators; the tube formula for a tile of inradius $1 / x$ is

$$
\gamma_{q}(x, \varepsilon)= \begin{cases}\kappa_{q 0}(\varepsilon) x^{0}+\kappa_{q 1}(\varepsilon) x^{1}=\alpha_{q}\left(-\varepsilon^{2}+2 \varepsilon x\right), & \varepsilon \leq 1 / x \\ \kappa_{q 2}(\varepsilon) x^{2}=\alpha_{q} x^{2}, & \varepsilon \geq 1 / x\end{cases}
$$

where $\alpha_{p}:=5 \cot \frac{3}{10} \pi$ and $\alpha_{t}:=\left(\cot \frac{\pi}{5}\right) /\left(1-\tan ^{2} \frac{\pi}{5}\right)$. Since $G_{2}, \ldots, G_{6}$ are congruent, we will apply Corollary 8.7 to a triangle $G_{t}$ and multiply by 5 before adding it to the result of applying Corollary 8.7 to the pentagon $G_{p}$. For the pentagon and the triangle, we have $\kappa_{0}=-\alpha_{q}, \kappa_{1}=2 \alpha_{q}$, and $\kappa_{2}=-\alpha_{q}$.

The tubular zeta function of $\mathcal{P}$ is

$$
\zeta_{\mathcal{P}}(\varepsilon, s)=\sum_{q=1}^{6} \frac{\alpha_{q} g_{q}^{s}}{1-5 \cdot r^{-s}}\left(-\frac{1}{s}+\frac{2}{s-1}-\frac{1}{s-2}\right) \varepsilon^{2-s},
$$

and the tube formula for $\mathcal{P}$ is

$$
V_{\mathcal{P}}(\varepsilon)=\frac{\alpha_{p}}{\log r^{-1}} \sum_{n \in \mathbb{Z}} g_{p}^{2}\left(-\frac{1}{D+i n \mathbf{p}}+\frac{2}{D-1+i n \mathbf{p}}-\frac{1}{D-2+i n \mathbf{p}}\right)\left(\frac{\varepsilon}{g_{p}}\right)^{2-D-i n \mathbf{p}}
$$




$$
\begin{aligned}
& +\frac{5 \alpha_{t}}{\log r^{-1}} \sum_{n \in \mathbb{Z}} g_{t}^{2}\left(-\frac{1}{D+i n \mathbf{p}}+\frac{2}{D-1+i n \mathbf{p}}-\frac{1}{D-2+i n \mathbf{p}}\right)\left(\frac{\varepsilon}{g_{t}}\right)^{2-D-i n \mathbf{p}} \\
& +\left[\left(\frac{\alpha_{p}}{4}+\frac{5 \alpha_{t}}{4}\right) \varepsilon^{2}+\frac{\left(2 \alpha_{p} g_{q}+10 \alpha_{p} g_{q} r\right) r}{r-5} \varepsilon\right]
\end{aligned}
$$

with $r=\phi^{-2}, \alpha_{p}=5 \cot \frac{3}{10} \pi, \alpha_{t}=\left(\cot \frac{\pi}{5}\right) /\left(1-\tan ^{2} \frac{\pi}{5}\right), g_{p}=\frac{\phi^{2}}{2} \tan \frac{3}{10} \pi, g_{t}=\frac{\phi^{3}}{2} \tan \frac{\pi}{5}$, $D=\log _{1 / r} 5$ and $\mathbf{p}=\frac{2 \pi}{\log r^{-1}}$.

Remark 9.4 Much as in the case of fractal strings where $d=1$ (see [30], Sect. 8.4.2), it follows from Theorem 8.3 that for a lattice self-similar tiling $\mathcal{T}$, each line of simple complex dimensions $\beta+i n \mathbf{p}$ gives rise to a function which consists of a multiplicatively periodic function times $\varepsilon^{d-\beta}$. Here, $\beta$ is some real constant and $\mathbf{p}=2 \pi / \log r^{-1}$ is the oscillatory period of $\mathcal{T}$. Consequently, since the scaling complex dimensions with real part $D$ are always simple, the tube formula for each lattice tiling in this section has the form

$$
V_{\mathcal{T}}(\varepsilon)=h\left(\log _{r^{-1}}\left(\varepsilon^{-1}\right)\right) \varepsilon^{d-\beta}+P(\varepsilon),
$$

where $h$ is an additively periodic function of period 1 and $P$ is a polynomial in $\varepsilon$. For instance, the periodic function appearing in the tube formula (9.9) for the Koch tiling $\mathcal{K}$ of Example 9.2 has the following Fourier expansion:

$$
h(u)=\frac{g}{\log 3} \sum_{n \in \mathbb{Z}} g^{i n \mathbf{p}}\left(-\frac{1}{D+i n \mathbf{p}}+\frac{2}{D-1+i n \mathbf{p}}-\frac{1}{D-2+i n \mathbf{p}}\right) e^{2 \pi i n u},
$$

where $g=\sqrt{3} / 18, D=\log _{3} 4, r=1 / \sqrt{3}$, and $\mathbf{p}=4 \pi / \log 3$. We note that multiplicatively periodic terms appear frequently in the mathematics and physics literature. See, for example, the relevant references given in Sect. 1.5, Sect. 2.7, Sect. 6.6, and Sect. 12.5 of [30].

\section{Some Remarks on the Results in This Paper}

In this last section, we briefly comments on several consequences and possible extensions of our main results.

Remark 10.1 The monograph [30] proposes a new definition of a fractal as "an object with nonreal complex dimensions that have a positive real part". With respect to this definition, the present work confirms the fractal nature of all the examples discussed in Sect. 9, and more generally, of all self-similar tilings considered in this paper.

Remark 10.2 Our results for tilings shed new light on the (1-dimensional) tube formula for fractal strings (1.11). The origin of the previously mysterious linear term $\left\{2 \varepsilon \zeta_{\mathcal{L}}(0)\right\}$ (see (6.16)) is now seen to come from a monophase formula for the unit interval, akin to (5.2). This is discussed further in Sect. 8.2. In fact, all terms coming from the second sum of the extended distributional formula of Theorem 6.4 are now understood to be related to a pluriphase formula. This reveals a geometric interpretation and allows the two sums to be naturally combined, as seen in (7.26) and (8.10). 
Remark 10.3 Many classical fractal curves are attractors of more than one self-similar system. For example, the Koch curve discussed in Sect. 9.2 is also the attractor of a system of four similarity transformations of $\mathbb{R}^{2}$, each with scaling ratio $r=\frac{1}{3}$. In this particular example, changes in the scaling zeta function produce a different set of complex dimensions. In fact, we obtain a subset of the original complex dimensions: $\left\{\log _{3} 4+\stackrel{\circ}{i n} \mathbf{p}: n \in\right.$ $\mathbb{Z}, \mathbf{p}=4 \pi / \log 3\}$. This has a natural geometric interpretation which is to be discussed in later work. In particular, it would be desirable to determine precisely which characteristics remain invariant between different tilings which are so related.

Remark 10.4 The two formulas (1.6) and (1.10) initially appear to be measuring very different things, but this is misleading. If one considers the example of the Sierpinski tiling (discussed in Sect. 9.3), then it is immediately apparent that the exterior $\varepsilon$-neighbourhood of the Sierpinski gasket is, in fact, equal to the union of the inner $\varepsilon$-neighbourhood of the tiling and the exterior $\varepsilon$-neighbourhood of the largest triangle. With $C_{0}$ as in Fig. 4 ,

$$
\operatorname{vol}_{2}\left(\mathcal{S G}_{\varepsilon}^{\text {ext }}\right)=V_{\mathcal{S G}}(\varepsilon)+\operatorname{vol}_{2}\left(C_{0}\right)
$$

However, things do not always work out so neatly, as the example of the Koch tiling shows; see Sect. 9.2. In the forthcoming paper [34], precise conditions are given for equality to hold as in (10.1). This allows one to use results of the present paper to compute explicit tube formulas for a large family of self-similar sets, including the Sierpinski gasket and carpet; see Remark 9.3.

Despite the fact that the tube formula for a self-similar tiling may differ from the tube formula for the corresponding self-similar set, it still gives us valuable information about self-similar geometries (and their associated dynamical systems). Indeed, we can define the complex dimensions of a given self-similar set in $\mathbb{R}^{d}$ to be those of the self-similar tiling canonically associated to it (as in [32]). This is motivated by focusing on the dynamics of the self-similar system, rather than looking directly at the set. For an example, see Sect. 9.2, especially Remark 9.1.

Remark 10.5 Recall that Weyl's celebrated formula [41] expresses the leading asymptotics of the eigenvalue counting function of the (Dirichlet) Laplacian on a $d$-dimensional compact Riemann manifold $M$ in terms of the volume of $M$ and its dimension $d$; see, e.g., [30, Sect. 12.5 and Appendix B]. An analogue of this formula exists for certain manifolds with fractal boundaries (for example, very irregular bounded open sets in $\mathbb{R}^{d}$ ), and in that case, the corresponding error term can be expressed in terms of the Minkowski dimension of the boundary; see [17-19], along with [30, Sect. 12.5.1 and Sect. 12.5.2] and the relevant references therein. For a fractal spray satisfying suitable hypotheses, we should be able to use the framework and the results of the present paper in order to obtain a full spectral asymptotic expansion (not just the leading term) for the Laplacian, expressed in terms of the underlying visible complex dimensions. See [30, Sects. 6.3-6.5] for the 1-dimensional case, and [30, Sect. 6.6] for an example in the case of a of self-similar fractal spray: the Sierpinski drum. We hope to elaborate on this remark in a later work.

Remark 10.6 (Note added in proof) It turns out that a little more care is needed for the results concerning Minkowski measurability to ensure that $\lim _{\varepsilon \rightarrow 0^{+}} V(\mathcal{T}, \varepsilon) \varepsilon^{-(d-D)}$ (or its counterpart in the lattice case, the average Minkowski content) does not vanish in (8.3), and that the corresponding term in the explicit formula dominates the remaining ones. This issue was discovered during collaboration with Steffen Winter on the forthcoming paper [24], and 
so it will be discussed in full detail in a sequel to that paper. For example, a sufficient condition for Corollary 8.5 to hold is that $D>d-1$ and

$$
\sum_{k=0}^{d-1} \frac{g^{D-k}}{D-k}(d-k) \kappa_{k}(G) \neq 0,
$$

where $g$ is the inradius of the monophase generator $G$ and $\kappa_{k}(G)$ is as in Definition 5.2.

Acknowledgements The authors wish to thank Martina Zähle for several helpful discussions on geometric measure theory and for bringing Ref. [13] to our attention. Additionally, the authors would like to thank Steffen Winter for many helpful discussions and suggestions, and for finding mistakes in an earlier version of this paper. Steffen also suggested the term "monophase" (the authors had originally used the term "diphase" in an earlier draft of this paper).

\section{Appendix A: The Definition and Properties of $\zeta_{\mathcal{T}}$}

In this appendix, we confirm some basic properties of the tubular zeta function $\zeta_{\mathcal{T}}$ of a fractal spray $\mathcal{T}$. However, we first require some facts about Mellin transformation. If $\psi \in$ $\mathbb{D}=C_{c}^{\infty}(0, \infty)$, it is elementary to check that for every $s \in \mathbb{C}$, the Mellin transform $\tilde{\psi}(s)$ is given by the well-defined integral (6.14) and satisfies $|\tilde{\psi}(s)| \leq|\tilde{\psi}|(\operatorname{Re} s)<\infty$. We will need additional estimates in what follows. We also use the forthcoming fact that $\tilde{\psi}(s)$ is an entire function; see Corollary A.4.

Throughout Appendix A, we assume that the hypotheses of Theorem 7.4 are satisfied.

Lemma A.1 Suppose that $S \subseteq \mathbb{C}$ is horizontally bounded, so inf $S:=\inf _{S} \operatorname{Re} s$ and $\sup S:=$ $\sup _{S} \operatorname{Re} s$ are finite. Let $K$ be a compact interval containing the support of $\psi \in C_{c}^{\infty}(0, \infty)$. Then there is a constant $c_{K}>0$ depending only on $K$ such that

$$
\sup _{s \in S}|\tilde{\psi}(s)| \leq c_{K}\|\psi\|_{\infty}
$$

In particular, $\tilde{\psi}(s)$ is always uniformly bounded on any screen $S$ as in Definition 6.1.

Proof Let $K$ be a compact interval containing the support of $\psi$. Since

$$
\left|x^{s-1}\right|=x^{\operatorname{Re} s-1} \leq \begin{cases}x^{\sup S-1}, & x \geq 1, \\ x^{\text {inf } S-1}, & 0<x<1,\end{cases}
$$

one can define a bound

$$
b_{K}:=\sup _{x \in K} \max \left\{x^{\sup S-1}, x^{\inf S-1}\right\} .
$$

Note that $b_{K}$ is finite because the function $x \mapsto \max \left\{x^{\sup S-1}, x^{\inf S-1}\right\}$ is continuous on the compact set $K$, and hence is bounded. Then we use (A.2) to bound $\tilde{\psi}$ as follows:

$$
\begin{aligned}
|\tilde{\psi}(s)| & \leq \int_{0}^{\infty}\left|x^{s-1}\right| \cdot|\psi(x)| d x \\
& =\int_{K} x^{\operatorname{Re} s-1}|\psi|(x) d x=|\tilde{\psi}|(\operatorname{Re} s) \\
& \leq b_{K}\|\psi\|_{\infty} \cdot \operatorname{vol}_{1}(K) .
\end{aligned}
$$


Remark A.2 The exact counterpart of Lemma A.1 holds if $\tilde{\psi}(s)$ is replaced by a translate $\tilde{\psi}\left(s-s_{0}\right)$, for any $s_{0} \in \mathbb{C}$. Therefore, under the same assumptions as in Lemma A.1, we have

$$
\sup _{s \in S}\left|\tilde{\psi}\left(s-s_{0}\right)\right| \leq c_{K, s_{0}}\|\psi\|_{\infty}
$$

where $c_{K, s_{0}}:=b_{K, s_{0}} \cdot \operatorname{vol}_{1}(K)$, and

$$
b_{K, s_{0}}:=\sup _{x \in K} \max \left\{x^{\sup S-\operatorname{Re} s_{0}-1}, x^{\inf S-\operatorname{Re} s_{0}-1}\right\}<\infty .
$$

In particular, for any compact interval $K$ containing the support of $\psi$, and for each fixed integer $k \geq 0$,

$$
\sup _{s \in S}|\tilde{\psi}(s-d+k+1)| \leq c_{K, k}\|\psi\|_{\infty}
$$

where $c_{K, k}$ is a finite and positive constant.

Lemma A.3 Let $(X, \mu)$ be a measure space. Define an integral transform by $F(s)=$ $\int_{X} f(x, s) d \mu(x)$ where

$$
|f(x, s)| \leq G(x), \quad \text { for some } G \in L^{1}(X, \mu),
$$

for $\mu$-a.e. $x \in X$, and for all $s$ in some neighbourhood of $s_{0} \in \mathbb{C}$. If the function $s \mapsto f(x, s)$ is holomorphic for $\mu$-a.e. $x \in X$, then $F(s)$ is well-defined and holomorphic at $s_{0}$.

The proof is a well-known application of Lebesgue's Dominated Convergence Theorem. We use Lemma A.3 to obtain the following corollary, which is used to prove Theorem 7.4 and Theorem A.7.

Corollary A.4 For $\psi \in C_{c}^{\infty}(0, \infty), \tilde{\psi}(s)$ is entire.

Proof Fix $s_{0} \in \mathbb{C}$. If $s$ is in a compact neighbourhood of $s_{0}$, then $\operatorname{Re} s$ is bounded, say by $\alpha \in \mathbb{R}$. Then for almost every $x>0$,

$$
\left|x^{s-1} \psi(x)\right| \leq x^{\alpha-1}\|\psi\|_{\infty} \chi_{\psi},
$$

where $\chi_{\psi}$ is the characteristic function of the compact support of $\psi$. Upon application of Lemma A.3, one deduces that $\psi$ is holomorphic at $s_{0}$.

Caution: Corollary A.4 does not combine with Lemma A.1 to imply that $\tilde{\psi}$ is constant; indeed, Liouville's Theorem does not apply here because $s$ is restricted to the screen $S$ in Lemma A.1.

Definition A.5 For $T(\varepsilon, s)$ to be a weakly meromorphic distribution-valued function on $W$, there must exist (i) a discrete set $\mathcal{P}_{T} \subseteq \mathbb{C}$, and (ii) for each $\omega \in \mathcal{P}_{T}$, an integer $n_{\omega}<\infty$, such that $\Psi(s)=\langle T(\varepsilon, s), \psi(\varepsilon)\rangle$ is a meromorphic function of $s \in W$, and each pole $\omega$ of $\Psi$ lies in $\mathcal{P}_{T}$ and has multiplicity at most $n_{\omega}$. 
To say that the distribution-valued function $T: W \rightarrow \mathbb{D}^{\prime}$ given by $s \mapsto T(\varepsilon, s)$ is (strongly) meromorphic means that, as a $\mathbb{D}^{\prime}$-valued function, it is truly a meromorphic function, in the sense of the proof of Lemma A.6. Recall that we are working with the space of distributions $\mathbb{D}^{\prime}$, defined as the dual of the space of test functions $\mathbb{D}=C_{c}^{\infty}(0, \infty)$.

Lemma A.6 If $T$ is a weakly meromorphic distribution-valued function, then it is a (strongly) meromorphic distribution-valued function.

Proof For $\omega \notin \mathcal{P}_{T}$, note that as $s \rightarrow \omega$,

$$
\frac{T(\varepsilon, s)-T(\varepsilon, \omega)}{s-\omega}
$$

converges to a distribution (call it $T^{\prime}(\varepsilon, \omega)$ ) in $\mathbb{D}^{\prime}$, by the Uniform Boundedness Principle for a topological vector space such as $\mathbb{D}$; see [35], Theorem 2.5 and Theorem 2.8. Hence, the $\mathbb{D}^{\prime}$-valued function $T$ is holomorphic at $\omega$.

For $\omega \in \mathcal{P}_{T}$, apply the same argument to

$$
\lim _{s \rightarrow \omega} \frac{1}{\left(n_{\omega}-1\right) !}\left(\frac{d}{d s}\right)^{n_{\omega}-1}\left((s-\omega)^{n_{\omega}} T(\varepsilon, s)\right),
$$

which must therefore define a distribution, i.e., exist as an element of $\mathbb{D}^{\prime}$. Thus $T$ is truly a meromorphic function with values in $\mathbb{D}^{\prime}$, and with poles contained in $\mathcal{P}_{T}$.

Theorem A.7 Under the hypotheses of Theorem 7.4 or Theorem 8.3, the tubular zeta function of a fractal spray or tiling

$$
\zeta_{\mathcal{T}}(\varepsilon, s)=\varepsilon^{d-s} \zeta_{\mathfrak{s}}(s) \sum_{k=0}^{d} \frac{g^{s-k}}{s-k} \kappa_{k}
$$

is a distribution-valued (strongly) meromorphic function on $W$, with poles contained in $\mathcal{D}_{\mathcal{T}}$.

Proof Let $\mathcal{P}_{T}=\mathcal{D}_{\mathcal{T}}$ and note that

$$
\begin{aligned}
\left\langle\zeta_{\mathcal{T}}(\varepsilon, s), \psi(\varepsilon)\right\rangle & =\zeta_{\mathfrak{F}}(s) \sum_{k=0}^{d} \frac{g^{s-k}}{s-k} \kappa_{k} \int_{0}^{\infty} \varepsilon^{d-s} \psi(\varepsilon) d \varepsilon \\
& =\zeta_{\mathfrak{s}}(s) \tilde{\psi}(d-s+1) \sum_{k=0}^{d} \frac{g^{s-k}}{s-k} \kappa_{k} .
\end{aligned}
$$

By Corollary A.4, this is a finite sum of meromorphic functions and hence meromorphic on $W$, for any test function $\psi$. Applying Lemma A.6, one sees that $\zeta_{\mathcal{T}}$ is a meromorphic function with values in $\mathbb{D}^{\prime}$.

Remark A.8 Note that for each $\psi \in \mathbb{D}$, the poles of the $\mathbb{C}$-valued function

$$
s \mapsto\left\langle\zeta_{\mathcal{T}}(\varepsilon, s), \psi(\varepsilon)\right\rangle
$$

are contained in $\mathcal{D}_{\mathcal{T}}$. Further, if $m_{\omega}$ is the multiplicity of $\omega \in \mathcal{D}_{\mathcal{T}}$ as a pole of $\zeta_{\mathfrak{F}}(s)$, then the multiplicity of $\omega$ as a pole of (A.12) is bounded by $m_{\omega}+1$. 
Corollary A.9 The residue of $\zeta_{\mathcal{T}}$ at a pole $\omega \in \mathcal{D}_{\mathcal{T}}$ is a well-defined distribution.

Proof This follows immediately from the second part of the proof of Lemma A.6, with $\mathcal{P}_{T}=\mathcal{D}_{\mathcal{T}}$.

Corollary A.10 The sum of residues appearing in Theorem 7.4 and Theorem 8.3 is distributionally convergent, and is thus a well-defined distribution.

Proof In view of the proof of Theorem A.7, this comes by applying the Uniform Boundedness Principle to an appropriate sequence of partial sums, in a manner similar to the proof of Lemma A.6. Again, see [30, Remark 5.21].

\section{Appendix B: The Error Term and Its Estimate}

In this appendix, we give the promised proof of the expression for the error term (7.5) and its estimate (7.6), as stated in Theorem 7.4. Throughout Appendix B, we assume that the hypotheses of Theorem 7.4 are satisfied. First, we require a definition.

Definition B.1 (Primitives of Distributions) Let $T_{\eta}$ be a distribution defined by a measure as $\left\langle T_{\eta}, \psi\right\rangle:=\int_{0}^{\infty} \psi d \eta$. Then the $k^{\text {th }}$ primitive (or $k^{\text {th }}$ antiderivative) of $T_{\eta}$ is defined by $\left\langle T_{\eta}^{[k]}, \psi\right\rangle:=(-1)^{k}\left\langle T_{\eta}, \psi^{[k]}\right\rangle$, where $\psi^{[k]}$ is the $k^{\text {th }}$ primitive of $\psi \in C_{c}^{\infty}(0, \infty)$ that vanishes at $\infty$ together with all its derivatives. For $k \geq 1$, for example,

$$
\left\langle T_{\eta}^{[k]}, \psi\right\rangle=\int_{0}^{\infty} \int_{y}^{\infty} \frac{(x-y)^{k-1}}{(k-1) !} \psi(x) d x d \eta(y) .
$$

Theorem B.2 The Mellin transform of the $k^{\text {th }}$ primitive of a test function is given by $\widetilde{\psi^{[k]}}(s)=\tilde{\psi}(s+k) \xi_{k}(s)$, where $\xi_{k}$ is the meromorphic function

$$
\xi_{k}(s):=\sum_{j=0}^{k-1} \frac{\left(\begin{array}{c}
k-1 \\
j
\end{array}\right)(-1)^{j}}{(k-1) !(s+j)} .
$$

Proof By direct computation,

$$
\begin{aligned}
\widetilde{\psi^{[k]}}(s) & =\int_{0}^{\infty} \varepsilon^{s-1} \int_{\varepsilon}^{\infty} \frac{(x-\varepsilon)^{k-1}}{(k-1) !} \psi(x) d x d \varepsilon \\
& =\frac{1}{(k-1) !} \int_{0}^{\infty} \int_{\varepsilon}^{\infty} \sum_{j=0}^{k-1}\left(\begin{array}{c}
k-1 \\
j
\end{array}\right) x^{k-1-j}(-\varepsilon)^{j} \varepsilon^{s-1} \psi(x) d x d \varepsilon \\
& =\sum_{j=0}^{k-1} \frac{\left(\begin{array}{c}
k-1 \\
j
\end{array}\right)(-1)^{j}}{(k-1) !} \int_{0}^{\infty} \int_{\varepsilon}^{\infty} x^{k-1-j} \varepsilon^{s+j-1} \psi(x) d x d \varepsilon \\
& =\sum_{j=0}^{k-1} \frac{\left(\begin{array}{c}
k-1 \\
j
\end{array}\right)(-1)^{j}}{(k-1) !} \int_{0}^{\infty} x^{k-1-j} \psi(x) \int_{0}^{x} \varepsilon^{s+j-1} d \varepsilon d x
\end{aligned}
$$




$$
\begin{aligned}
& =\sum_{j=0}^{k-1} \frac{\left(\begin{array}{c}
k-1 \\
j
\end{array}\right)(-1)^{j}}{(k-1) !(s+j)} \int_{0}^{\infty} x^{s+k-1} \psi(x) d x \\
& =\tilde{\psi}(s+k) \xi_{k}(s) .
\end{aligned}
$$

Again, the formula (B.2) for $\xi_{k}$ is valid for $\operatorname{Re} s>k$ by (B.3), but then extends to being valid for all $s \in \mathbb{C}$ by meromorphic continuation.

Corollary B.3 We also have $\left|\widetilde{\psi^{[k]}}(s)\right| \leq\left|\tilde{\psi}(s+k) \xi_{k}(s)\right|$.

Remark B.4 For $s \in \mathbb{C}, t=\operatorname{Im} s$, and $c_{\xi}>0$, we also have

$$
\left|\xi_{k}(s)\right| \leq \frac{c_{\xi}}{|t|^{k}}
$$

We are now in a position to provide the proofs previously promised.

Theorem B.5 As stated in (7.5) of Theorem 7.4, the error term is given by

$$
\mathcal{R}(\varepsilon)=\frac{1}{2 \pi \stackrel{\circ}{0}} \int_{S} \zeta_{\mathcal{T}}(\varepsilon, s) d s,
$$

and is a well-defined distribution.

Proof Applying (6.14) to (7.24) for $k=0, \ldots, d$ gives $^{12}$

$$
\langle\mathcal{R}, \varphi\rangle_{k}=\frac{1}{2 \pi \AA} \int_{S} \frac{g^{s-k}}{s-k} \zeta_{\mathfrak{s}}(s) \kappa_{k} \int_{0}^{\infty} \varepsilon^{d-s} \psi(\varepsilon) d \varepsilon d s .
$$

To see that this gives a well-defined distribution $\mathcal{R}$, we apply the descent method, as described in [30], Remark 5.20. The first step is to show that $\left\langle\mathcal{R}^{[k]}, \psi\right\rangle_{k}$ is a well-defined distribution for sufficiently large $k$; specifically, for any integer $k>\varpi$, where $\varpi$ is the order of languidity, as in Definition 6.2. Note that we can break the integral along the screen $S$ into two pieces and work with each separately:

$$
\begin{aligned}
\left\langle\mathcal{R}^{[k]}, \psi\right\rangle_{k}= & \frac{(-1)^{k}}{2 \pi \AA} \int_{|\operatorname{Im} s|>1} \frac{g^{s-k}}{s-k} \zeta_{\mathfrak{s}}(s) \kappa_{k} \int_{0}^{\infty} \varepsilon^{d-s} \psi^{[k]}(\varepsilon) d \varepsilon d s \\
& +\frac{(-1)^{k}}{2 \pi \AA} \int_{|\operatorname{Im} s| \leq 1} \frac{g^{s-k}}{s-k} \zeta_{\mathfrak{s}}(s) \kappa_{k} \int_{0}^{\infty} \varepsilon^{d-s} \psi^{[k]}(\varepsilon) d \varepsilon d s .
\end{aligned}
$$

Here and throughout the rest of this appendix, it is understood that such integrals (as in (B.7)-(B.8)) are for $s \in S$. Since the screen avoids the integers $0, \ldots, d$ by assumption, the quantity $|s-k|$ is bounded away from 0 . Since the screen avoids the poles of $\zeta_{\mathfrak{s}}$ by

\footnotetext{
${ }^{12}$ In the proof of Theorem 7.4, the quantity (B.6) was denoted by $\left\langle\mathcal{R}_{k}, \psi\right\rangle$, so that $\mathcal{R}$ could easily be written (formally) as a function in (7.27). For clarity, since we work with test functions, this quantity is instead denoted by $\langle\mathcal{R}, \psi\rangle_{k}$ throughout this proof.
} 
hypothesis, $\zeta_{\mathfrak{F}}(s)$ is continuous on the compact set $\{s \in S:|\operatorname{Im} s| \leq 1\}$. Therefore, it is clear that (B.8) is a well-defined integral. We focus now on (B.7):

$$
\begin{aligned}
& \left|\frac{\kappa_{k}}{2 \pi \AA} \int_{|\operatorname{Im} s|>1} \frac{g^{s-k}}{s-k} \zeta_{\mathfrak{s}}(s) \int_{0}^{\infty} \varepsilon^{d-s} \psi^{[k]}(\varepsilon) d \varepsilon d s\right| \\
& \quad \leq \frac{\kappa_{k}}{2 \pi} \int_{\operatorname{Im} s>1}\left|g^{s-k} \frac{\zeta_{\mathfrak{s}}(s)}{s-k}\right| \cdot\left|\widetilde{\psi^{[k]}}(s-d+1)\right| d s \\
& \quad \leq c_{1} \int_{1}^{\infty}|t|^{M-1} \cdot|\tilde{\psi}(s-d+k+1)| \cdot\left|\xi_{k}(s-d+1)\right| d t \\
& \quad \leq c_{1} \int_{1}^{\infty} c_{k}|t|^{M-1} \cdot c_{K}\|\psi\|_{\infty} \cdot \frac{c_{\xi}}{|t|^{k}} d t, \\
& \quad=C\|\psi\|_{\infty} \int_{1}^{\infty}|t|^{M-1-k} d t,
\end{aligned}
$$

which is clearly convergent for $k>M$. The second inequality in (B.9) comes by condition L2 of Definition 6.2. Also, recall (from the remark just after the statement of Lemma A.1) that for $s \in S$, the real part of $s$ is given by some function $f$ which is Lipschitz (cf. Definition 6.1), and hence is almost everywhere differentiable and has a bounded derivative (where it exists) on the support of $\psi$. The third comes by inequality (A.5) of Remark A.2, along with Remark B.4. This establishes the validity of $\left\langle\mathcal{R}^{[k]}, \psi\right\rangle_{k}$ and thus shows that $\mathcal{R}^{[k]}$ defines a linear functional on $\mathbb{D}$.

To check that the action of $\mathcal{R}^{[k]}$ is continuous on $\mathbb{D}$, let $\psi_{n} \rightarrow 0$ in $\mathbb{D}$, so that there is a compact set $K$ which contains the support of every $\psi_{n}$, and $\left\|\psi_{n}\right\|_{\infty} \rightarrow 0$. Then

$$
\left|\left\langle\mathcal{R}^{[k]}, \psi_{n}\right\rangle\right| \leq C \cdot\left|\tilde{\psi}_{n}(s-d+k+1)\right| \leq c_{K}\left\|\psi_{n}\right\|_{\infty} \stackrel{n \rightarrow \infty}{\longrightarrow} 0,
$$

by following (B.9) and then applying Lemma A.1, along with its extensions as stated in Remark A.2. Thus, $\mathcal{R}^{[k]}$ is a well-defined distribution. If we differentiate it distributionaly $k$ times, we obtain $\mathcal{R}$. This shows that $\mathcal{R}$ is a well-defined distribution and concludes the proof.

Before finally checking the error estimate, we define what is meant by the expression $T(x)=O\left(x^{\alpha}\right)$ as $x \rightarrow \infty$, when $T$ is a distribution.

Definition B.6 When $\mathcal{R}(x)=O\left(x^{\alpha}\right)$ as $x \rightarrow \infty$ (as in (6.13)), we say as in [30], Sect. 5.4.2, that $\mathcal{R}$ is of asymptotic order at most $x^{\alpha}$ as $x \rightarrow \infty$. To understand this expression, first define

$$
\psi_{a}(x):=\frac{1}{a} \psi\left(\frac{x}{a}\right)
$$

for $a>0$ and for any test function $\psi$. Then " $\mathcal{R}(x)=O\left(x^{\alpha}\right)$ as $x \rightarrow \infty$ " means that

$$
\left\langle\mathcal{R}, \psi_{a}\right\rangle=O\left(a^{\alpha}\right), \quad \text { as } a \rightarrow \infty,
$$

for every test function $\psi$. The implied constant may depend on $\psi$. Similarly, " $\mathcal{R}(x)=$ $O\left(x^{\alpha}\right)$ as $x \rightarrow 0^{+}$" (as in (6.18) and (7.6)) is defined to mean that

$$
\left\langle\mathcal{R}, \psi_{a}\right\rangle=O\left(a^{\alpha}\right), \quad \text { as } a \rightarrow 0^{+},
$$

for every test function $\psi$. 
Theorem B.7 (Error Estimate) As stated in Theorem 7.4, the error term $\mathcal{R}(\varepsilon)$ in (B.5) is estimated by

$$
\mathcal{R}(\varepsilon)=O\left(\varepsilon^{d-\sup S}\right), \quad \text { as } \varepsilon \rightarrow 0^{+} .
$$

Proof As in the proof of Theorem B.5, we use the descent method and begin by splitting the integral into two pieces. Since

$$
\left\langle\mathcal{R}^{[k]}, \psi_{a}\right\rangle=(-1)^{k}\left\langle\mathcal{R},\left(\psi_{a}\right)^{[k]}\right\rangle,
$$

we work with

$$
\begin{aligned}
\left\langle\mathcal{R},\left(\psi_{a}\right)^{[k]}\right\rangle_{k}= & \frac{\kappa_{k}}{2 \pi \AA} \int_{|\operatorname{Im} s|>1} \frac{g^{s-k}}{s-k} \zeta_{\mathfrak{s}}(s) \int_{0}^{\infty} \varepsilon^{d-s}\left(\psi_{a}\right)^{[k]}(\varepsilon) d \varepsilon d s \\
& +\frac{\kappa_{k}}{2 \pi \AA} \int_{|\operatorname{Im} s| \leq 1} \frac{g^{s-k}}{s-k} \zeta_{\mathfrak{s}}(s) \int_{0}^{\infty} \varepsilon^{d-s}\left(\psi_{a}\right)^{[k]}(\varepsilon) d \varepsilon d s .
\end{aligned}
$$

The $k^{\text {th }}$ primitive of $\psi_{a}$ is given by

$$
\left(\psi_{a}\right)^{[k]}(\varepsilon)=\int_{\varepsilon}^{\infty} \frac{(u-\varepsilon)^{k-1}}{(k-1) !} \frac{1}{a} \psi\left(\frac{u}{a}\right) d u=\int_{\varepsilon / a}^{\infty} \frac{(a u-\varepsilon)^{k-1}}{(k-1) !} \psi(u) d u .
$$

By following the same calculations as in Theorem B.2, one observes that

$$
\begin{aligned}
& \left|\int_{0}^{\infty} \frac{\varepsilon^{d-s}}{s-k} \int_{\varepsilon / a}^{\infty} \frac{(a u-\varepsilon)^{k-1}}{(k-1) !} \psi(u) d u d \varepsilon\right| \\
& \quad=\left|\int_{0}^{\infty} \int_{0}^{a u} \frac{\sum^{k-1}}{s-k} \sum_{j=0}^{\left(\begin{array}{c}
k-1 \\
j
\end{array}\right)(-1)^{j}}(a u)^{k-1-j} \varepsilon^{d-s+j} \psi(u) d \varepsilon d u\right| \\
& \quad \leq \frac{1}{|s-k|} \sum_{j=0}^{k-1} \frac{\left(\begin{array}{c}
k-1 \\
j
\end{array}\right)(-1)^{j}}{(k-1) !} \int_{0}^{\infty}\left|(a u)^{k-1-j} \psi(u)\right| \int_{0}^{a u}\left|\varepsilon^{d-s+j} d \varepsilon\right| d u \\
& \quad \leq \frac{c_{k}}{|s-k|} \xi_{k}(d-\operatorname{Re} s+1) \int_{0}^{\infty}(a u)^{k-1-j}(a u)^{d-\operatorname{Re} s+j+1}|\psi(u)| d u \\
& \quad=a^{d-\operatorname{Re} s+k} \frac{c_{k}}{|s-k|} \xi_{k}(d-\operatorname{Re} s+1)|\tilde{\psi}|(d-\operatorname{Re} s+k) .
\end{aligned}
$$

Using (B.4) for $\xi_{k}$ and (A.3) for $|\tilde{\psi}|$ (see Remark A.2), we bound (B.13) by

$$
\begin{aligned}
& \frac{c_{k}}{2 \pi} \int_{|\operatorname{Im} s|>1} a^{d-\operatorname{Re} s+k} \cdot \frac{\left|g^{s-k} \zeta_{\mathfrak{s}}(s)\right|}{|s-i|} \cdot \frac{c_{\psi}}{|t|^{k}} \cdot c_{K}\|\psi\|_{\infty} d s \\
& \quad \leq a^{d-\sup S+k}\left(C \int_{1}^{\infty}|t|^{M-1-k} d t\right)
\end{aligned}
$$

for any $0<a<1$, as in (B.9). Since the integral in (B.18) clearly converges for $k>M$, we have established the estimate for $\mathcal{R}^{[k]}$, along the part of the integral where $|\operatorname{Im} s|>1$. Recall that all our contour integrals are taken along the screen $S$. The proof for (B.13), where 
$|\operatorname{Im} s|>1$, readily follows from the corresponding argument in the proof of Theorem B.5. Thus we have established that

$$
\left|\left\langle\mathcal{R}^{[k]}(\varepsilon), \psi_{a}(\varepsilon)\right\rangle\right| \leq a^{d-\sup S+k} c_{k}, \quad \text { for all } 0<a<1 .
$$

In (B.19)-(B.21), the constants $c_{k}$ may depend on the test function $\psi .^{13}$

By iterating the following calculation:

$$
\begin{aligned}
\left|\left\langle\mathcal{R}^{[k-1]}(\varepsilon), \psi_{a}(\varepsilon)\right\rangle\right| & =\left|\left\langle\mathcal{R}^{[k]}(\varepsilon),\left(\frac{1}{a} \psi\left(\frac{\varepsilon}{a}\right)\right)^{\prime}\right\rangle\right| \\
& =\left|\frac{1}{a}\left\langle\mathcal{R}^{[k]}(\varepsilon),\left(\psi^{\prime}\right)_{a}(\varepsilon)\right\rangle\right| \\
& \leq a^{d-\sup S+k-1} c_{k-1},
\end{aligned}
$$

one sees that

$$
\left|\left\langle\mathcal{R}(\varepsilon), \psi_{a}(\varepsilon)\right\rangle\right| \leq a^{d-\sup S} c_{0}, \quad \text { for all } 0<a<1 .
$$

By Definition B.6, this implies that $\mathcal{R}(\varepsilon)=O\left(\varepsilon^{d-\sup S}\right)$ as $\varepsilon \rightarrow 0^{+}$.

\section{References}

1. Berger, M., Gostiaux, B.: Differential Geometry: Manifolds, Curves and Surfaces. Springer, Berlin (1988) (English transl.)

2. Falconer, K.J.: Fractal Geometry-Mathematical Foundations and Applications. Wiley, Chichester (1990)

3. Falconer, K.J.: On the Minkowski measurability of fractals. Proc. Am. Math. Soc. 123, 1115-1124 (1995)

4. Federer, H.: Curvature measures. Trans. Am. Math. Soc. 93, 418-491 (1959)

5. Frantz, M.: Minkowski measurability and lacunarity of self-similar sets in $\mathbb{R}$. Proc. Symp. Pure Math. 72(1), 77-91 (2004)

6. Fu, J.H.G.: Tubular neighborhoods in Euclidean spaces. Duke Math. J. 52, 1025-1046 (1985)

7. Fu, J.H.G.: Curvature measures of subanalytic sets. Am. J. Math. 116, 819-880 (1994)

8. Gatzouras, D.: Lacunarity of self-similar and stochastically self-similar sets. Trans. Am. Math. Soc. 352, 1953-1983 (2000)

9. Gray, A.: Tubes, 2nd edn. Progress in Math., vol. 221. Birkhäuser, Boston (2004)

10. Hambly, B.M., Lapidus, M.L.: Random fractal strings: their zeta functions, complex dimensions and spectral asymptotics. Trans. Am. Math. Soc. 358, 285-314 (2006)

11. He, C.Q., Lapidus, M.L.: Generalized Minkowski content and the vibrations of fractal drums and strings. Math. Res. Lett. 3, 31-40 (1996)

12. He, C.Q., Lapidus, M.L.: Generalized Minkowski content, spectrum of fractal drums, fractal strings and the Riemann zeta-function. Mem. Am. Math. Soc. 127(608), 1-97 (1997)

13. Hug, D., Last, G., Weil, W.: A local Steiner-type formula for general closed sets and applications. Math. Z. 246, 237-272 (2004)

14. Hutchinson, J.E.: Fractals and self-similarity. Indiana Univ. Math. J. 30, 713-747 (1981)

15. Kigami, J.: Analysis on Fractals. Cambridge University Press, Cambridge (1999)

16. Klain, D.A., Rota, G.-C.: Introduction to Geometric Probability. Accademia Nazionale dei Lincei. Cambridge University Press, Cambridge (1999)

17. Lapidus, M.L.: Fractal drum, inverse spectral problems for elliptic operators and a partial resolution of the Weyl-Berry conjecture. Trans. Am. Math. Soc. 325, 465-529 (1991)

\footnotetext{
${ }^{13}$ Note that $c_{k-1}$ does not correspond to $c_{k}$ when $k$ is replaced by $k-1$; rather, $c_{k-1}$ depends on the support of $\psi^{\prime}$. The notation is just used to indicate the analogous roles the constants $c_{k}$ play.
} 
18. Lapidus, M.L.: Spectral and fractal geometry: From the Weyl-Berry conjecture for the vibrations of fractal drums to the Riemann zeta-function. In: Bennewitz, C. (ed.) Differential Equations and Mathematical Physics, Proc. Fourth UAB Internat. Conf., Birmingham, March 1990, pp. 151-182. Academic Press, New York (1992)

19. Lapidus, M.L.: Vibrations of fractal drums, the Riemann hypothesis, waves in fractal media, and the Weyl-Berry conjecture. In: Sleeman, B.D., Jarvis, R.J. (eds.) Ordinary and Partial Differential Equations vol. IV, Proc. Twelfth Internat. Conf., Dundee, Scotland, UK, June 1992. Pitman Research Notes in Math. Series, vol. 289, pp. 126-209. Longman Scientific and Technical, London (1993)

20. Lapidus, M.L., Maier, H.: The Riemann hypothesis and inverse spectral problems for fractal strings. J. Lond. Math. Soc. 52(2), 15-34 (1995)

21. Lapidus, M.L., Pearse, E.P.J.: A tube formula for the Koch snowflake curve, with applications to complex dimensions. J. Lond. Math. Soc. 74(2), 397-414 (2006). arXiv:math-ph/0412029, 2005

22. Lapidus, M.L., Pearse, E.P.J.: Tube formulas for self-similar fractals. In: Exner, P., Keating, J.P., Bristol, C., Kuchment, P., Sunada, T., Teplyaev, A. (eds.) Analysis on Graphs and Its Applications. Proc. of Symposia in Pure Mathematics, vol. 77, pp. 211-230. Am. Math. Soc., Providence (2008). arXiv: 0711.0173, 2007

23. Lapidus, M.L., Pearse, E.P.J., Winter, S.: Fractal curvature measures and local tube formulas (work in progress)

24. Lapidus, M.L., Pearse, E.P.J., Winter, S.: Pointwise tube formulas for fractal sprays and self-similar tilings with arbitrary generators. arXiv:1006.3807

25. Lapidus, M.L., Pomerance, C.: The Riemann-zeta function and the one-dimensional Weyl-Berry conjecture for fractal drums. Proc. Lond. Math. Soc. 66(3), 41-69 (1993)

26. Lapidus, M.L., Pomerance, C.: Counterexamples to the modified Weyl-Berry conjecture on fractal drums. Math. Proc. Camb. Philos. Soc. 119, 167-178 (1996)

27. Lapidus, M.L., van Frankenhuysen, M.: Fractal Geometry and Number Theory: Complex Dimensions of Fractal Strings and Zeros of Zeta Functions. Birkhäuser, Boston (2000)

28. Lapidus, M.L., van Frankenhuysen, M.: Complex dimensions of self-similar fractal strings and Diophantine approximation. J. Exp. Math. 12(1), 41-69 (2003)

29. Lapidus, M.L., van Frankenhuijsen, M.: Fractality, self-similarity and complex dimensions. Proc. Symp. Pure Math. 72(1), 349-372 (2004)

30. Lapidus, M.L., van Frankenhuijsen, M.: Fractal Geometry, Complex Dimensions and Zeta Functions: Geometry and Spectra of Fractal Strings. Springer Mathematical Monographs. Springer, New York (2006). (2nd revised and enlarged edition to appear in 2011)

31. Mattila, P.: Geometry of Sets and Measures in Euclidean Spaces (Fractals and Rectifiability). Cambridge University Press, Cambridge (1995)

32. Pearse, E.P.J.: Canonical self-affine tilings by iterated function systems. Indiana Univ. Math. J. 56(6), 3151-3169 (2007)

33. Pearse, E.P.J.: Complex dimensions of self-similar systems Ph.D. dissertation Univ. of California, Riverside, June 2006

34. Pearse, E.P.J., Winter, S.: Geometry of self-similar tilings. Rocky Mt. J. Math. (to appear). arXiv: 0811.2187

35. Rudin, W.: Functional Analysis, 2nd edn. McGraw-Hill, New York (1991)

36. Schneider, R.: Curvature measures of convex bodies. Ann. Mat. Pura Appl. 116(4), 101-134 (1978)

37. Schneider, R.: Convex Bodies: The Brunn-Minkowski Theory. Cambridge University Press, Cambridge (1993)

38. Stacho, L.L.: On curvature measures. Acta Sci. Math. 41, 191-207 (1979)

39. Tricot, C.: Curves and Fractal Dimensions. Springer, New York (1995)

40. Weyl, H.: On the volume of tubes. Am. J. Math. 61, 461-472 (1939)

41. Weyl, H.: Über die Abhängigkeit der Eigenschwingungen einer Membran von deren Begrenzung. J. Reine Angew. Math. 141, 1-11 (1912)

42. Zähle, M.: Integral and current representation of Federer's curvature measures. Arch. Math. 46, 557-567 (1986)

43. Zähle, M.: Curvatures and currents for unions of sets with positive reach. Geom. Dedic. 23, 155-171 (1987) 